\title{
Voronoi cell finite difference method for the diffusion operator on arbitrary unstructured grids
}

\author{
N. Sukumar*, \\ Department of Civil and Environmental Engineering, University of California, Davis, CA 95616, U.S.A.
}

\begin{abstract}
SUMMARY
Voronoi cells and the notion of natural neighbours are used to develop a finite difference method for the diffusion operator on arbitrary unstructured grids. Natural neighbours are based on the Voronoi diagram, which partitions space into closest-point regions. The Sibson and the Laplace (non-Sibsonian) interpolants which are based on natural neighbours have shown promise within a Galerkin framework for the solution of partial differential equations. In this paper, we focus on the Laplace interpolant with a two-fold objective: first, to unify the previous developments related to the Laplace interpolant and to indicate its ties to some well-known numerical methods; and secondly to propose a Voronoi cell finite difference scheme for the diffusion operator on arbitrary unstructured grids. A conservation law in integral form is discretized on Voronoi cells to derive a finite difference scheme for the diffusion operator on irregular grids. The proposed scheme can also be viewed as a point collocation technique. A detailed study on consistency is conducted, and the satisfaction of the discrete maximum principle (stability) is established. Owing to symmetry of the Laplace weight, a symmetric positive-definite stiffness matrix is realized which permits the use of efficient linear solvers. On a regular (rectangular or hexagonal) grid, the difference scheme reduces to the classical finite difference method. Numerical examples for the Poisson equation with Dirichlet boundary conditions are presented to demonstrate the accuracy and convergence of the finite difference scheme. Copyright (c) 2003 John Wiley \& Sons, Ltd.
\end{abstract}

KEY WORDS: natural neighbour; Sibson and Laplace interpolants; finite difference; finite volume; irregular grids; Poisson equation

\section{INTRODUCTION}

Numerical methods such as the finite element, finite difference, and finite volume schemes are widely used for the numerical modelling of physical phenomena such as elasticity, heat transfer, fluid flow, and electromagnetics. Finite elements are particularly useful when dealing with boundary-value problems on arbitrarily shaped geometries where $p$ - or $h$-adaptivity is desirable, whereas finite difference schemes are attractive when the domain geometry is regular and uniform nodal spacing suffices - the ease of constructing and implementing finite

\footnotetext{
*Correspondence to: N. Sukumar, Department of Civil and Environmental Engineering, One Shields Avenue, University of California, Davis, CA 95616. U.S.A.

†E-mail: nsukumar@ucdavis.edu

Received 11 January 2002

Revised 3 July 2002

Copyright (c) 2003 John Wiley \& Sons, Ltd.

Accepted 22 July 2002
} 
difference approximations on a regular grid is unmatched. Finite volume schemes are based on a volume integral formulation of the original partial differential equation (PDE) on a set of finite (control) volumes that partition the domain. This technique proves to be well-suited to deal with physical conservation laws (mass, momentum, and energy), such as those that arise in fluid dynamics, groundwater flow and contamination, and for shock-capturing applications. A recent development within the framework of Galerkin methods has been in the area of meshless methods [1]. With an aim towards alleviating the need to remesh in moving boundary and large deformation problems, there has been significant interest in these classes of methods for the solution of PDEs. In this paper, we use a data approximation technique recently adopted in meshless methods to construct a finite difference scheme on unstructured grids.

Classical finite difference schemes are typically based on regular grids, and hence are restricted to problem domains with regular geometry. Owing to the wide applicability of finite difference schemes for solving initial/boundary value problems in many areas of mathematical physics, there have been many contributions towards extending the methodology on irregular grids. Building on prior work due to Jensen [2], Liszka and Orkisz [3] proposed a generalized finite difference method (GFDM) on irregular grids. The central issue addressed in Reference [3] was the appropriate selection of the computational cell (star-shaped domain) that surrounds a node, so as to yield a well-conditioned linear system of equations. In Reference [3], a Taylor series approximation was used, whereas Breitkopf et al. [4] adopted moving least squares approximants [5] in the GFDM to derive the discrete form for the differential operators. Baty and Wolfe [6] have proposed a least-squares method for solving elliptic problems on arbitrary irregular grids.

The construction of monotone finite volume schemes for the diffusion operator on nonuniform grids has received significant attention [7-11]. The accurate modelling of the diffusion operator is required in heat conduction, groundwater flow simulations, electrostatics, integrated circuit development processes, and is also of importance in Lagrangian hydrodynamics, where mesh distortion is an issue. Süli [7], as well as Jones and Menzies [10], studied the convergence of cell-centred finite volume schemes on Cartesian product non-uniform grids, whereas Mishev [8] investigated the stability and error estimates of cell-centred finite volume schemes on Voronoi meshes. Hermeline [9] used degrees of freedom associated with a primary (Delaunay) mesh as well as a dual (Voronoi) mesh for an accurate approximation of the fluxes, and in Reference [11], the construction of positive (monotone) stencils for the Laplace operator is discussed.

The fundamental requirement for the solution of diffusion or convection-diffusion equations using finite element [12], finite volume methods [11], or finite differences is that the approximation must satisfy a discrete maximum principle - non-physical local extrema must not be present in the numerical solution, for otherwise spurious oscillations or even divergence can result. A scheme that satisfies a maximum principle is also known as a monotone scheme, and the sufficient condition for the same is that the stiffness matrix be an $M$-matrix (non-singular). This requirement stems from the fact that in a diffusion problem, the flow (fluid or heat) must travel from regions of higher potential to regions of lower potential, and hence the numerical approximation must mirror the same behaviour. Triangular finite elements are monotone if there are no obtuse angles in the Delaunay triangulation, but in $3 \mathrm{D}$, special modifications are required to yield a non-singular ( $M$-matrix) matrix [13]. The need to satisfy the discrete maximum principle renders the task of constructing monotone finite difference and finite volume schemes on arbitrary unstructured grids as non-trivial. 
Consider a domain $\Omega$ in $1 \mathrm{D}, 2 \mathrm{D}$, or $3 \mathrm{D}$, that is described by $M$ scattered nodes. In meshless Galerkin methods $[1,14]$, an approximant or interpolant is constructed solely on the basis of these nodes, without the use of any explicit connectivity (as in finite elements) information between these nodes. In a Galerkin procedure, a background mesh (Delaunay triangulation or quadrangulation in two-dimensions) is typically used for the numerical integration of the weak form. The meshless paradigm has provided new insights into the finite element method $[15,16]$, and also brought out the intimate link between scattered data approximation, computational geometry, and the numerical solutions of PDEs. The interested reader can refer to the review articles by Belytschko and co-workers [1] and $\mathrm{Li}$ and Liu [14] for a detailed discussion and comparison of different meshless and particle methods.

In most meshless methods, moving least squares (MLS) [5] approximants are used to construct the trial and test functions. The notable exceptions are those which are based on natural neighbours. The notion of natural neighbours that was introduced by Sibson $[17,18]$ is an attractive alternative to MLS approximants. The definition of natural neighbours is based on the Voronoi diagram of a nodal set. The Voronoi diagram [19] and its geometric dual, the Delaunay triangulation [20], are well-known geometric constructs in computational geometry. The Sibson [17,18] and the Laplace (non-Sibsonian) [21-26] interpolants, which are based on natural neighbours, have been used in a Galerkin framework to solve PDEs [27-39]. The ease of imposing essential boundary conditions as in finite elements, the computational efficiency of shape function computations, and the ability to construct a well-defined and robust approximation for irregular discretizations render these interpolants as very attractive for unstructured grids $[28,32]$.

In this paper, we focus on the Laplace interpolant with a two-fold objective: first, to unify the previous developments related to the Laplace interpolant and to indicate its ties to some well-known numerical methods; and secondly to construct a Voronoi cell finite difference method for the diffusion operator on unstructured grids. In Section 2, an extensive background on natural neighbour-based interpolants is provided. We trace the roots of the Laplace interpolant to studies on random lattices in the early 1980s [40-42], with its recent re-discovery in two distinct research communities: PDEs [21-23] and computational geometry [24-26]. In Section 3, links of the Laplace interpolant to other numerical methods and applications are discussed. A conservation law in integral form is discretized on Voronoi cells to derive a finite difference scheme for the diffusion operator on irregular grids (Section 3.1). A detailed theoretical analysis is conducted, in which consistency, solvability, and the satisfaction of the discrete maximum principle are studied (Sections 3.1.3-3.1.5). Owing to symmetry of the Laplace weight, a symmetric positive-definite stiffness matrix is realized which permits the use of efficient linear solvers. On a regular (rectangular or hexagonal) grid, the difference scheme reduces to the classical finite difference method (Section 3.1.6). In Section 4, numerical examples for the Poisson equation with Dirichlet boundary conditions are presented to demonstrate the accuracy and convergence of the finite difference scheme. The main results and conclusions from this study are discussed in Section 5.

\section{NATURAL NEIGHBOUR-BASED INTERPOLANTS}

Natural neighbours provide a means to associate neighbour relationships for scattered nodes by taking into account the relative spatial density and position of nodes, and do not rely on 
a Euclidean metric as in MLS-schemes. This leads to a more natural and robust approach to define interpolation schemes for non-uniform nodal discretizations. In the element-free Galerkin method [43], where MLS approximants are used, the support radius and choice of weight functions are free parameters to be set by the user. The appropriate choice and bounds for these parameters (especially in three-dimensions) so as to define a robust approximation for scattered nodes in $\Omega$ are still open questions; for a recent study in this direction, see Reference [44].

Consider a bounded domain $\Omega$ in $d$-dimensions $(d=1-3)$ that is described by a set $\mathbf{N}$ of $M$ scattered nodes: $\mathbf{N}=\left\{n_{1}, n_{2}, \ldots, n_{M}\right\}$. The Voronoi diagram $\mathscr{V}(\mathbf{N})$ of the set $\mathbf{N}$ is a subdivision of the domain into regions $V\left(n_{I}\right)$, such that any point in $V\left(n_{I}\right)$ is closer to node $n_{I}$ than to any other node $n_{J} \in N(J \neq I)$. The Voronoi diagram in essence partitions space into closest-point regions. The region $V\left(n_{I}\right)$ (Voronoi cell) for a node $n_{I}$ within the convex hull is a convex polygon (polyhedron) in $\mathbb{R}^{2}\left(\mathbb{R}^{3}\right)$ :

$$
V\left(n_{I}\right)=\left\{\mathbf{x} \in \mathbb{R}^{d}: d\left(\mathbf{x}, \mathbf{x}_{I}\right)<d\left(\mathbf{x}, \mathbf{x}_{J}\right) \forall J \neq I\right\}
$$

where $d\left(\mathbf{x}_{I}, \mathbf{x}_{J}\right)$ is the Euclidean distance between $\mathbf{x}_{I}$ and $\mathbf{x}_{J}$.

The Voronoi cell for each node is formed by the intersection of perpendicular half-spaces, whereas its dual, the Delaunay tessellation, is constructed by connecting nodes that have a common $(d-1)$-dimensional Voronoi facet. Given any nodal set $\mathbf{N}$, the Voronoi diagram is unique, whereas the Delaunay tessellation is not - a simple example is the triangulation of a square where choosing either diagonal leads to a Delaunay triangulation (see Figure 9(a)). In Figure 1(a), the Voronoi diagram and the Delaunay triangulation are shown for a nodal set consisting of seven nodes $(M=7)$. The Voronoi vertex and edge are also indicated in Figure 1(a). An important property of Delaunay triangles is the empty circumcircle criterion [45]—if $\mathscr{D} \mathscr{T}\left(n_{J}, n_{K}, n_{L}\right)$ is any Delaunay triangle of the nodal set $\mathbf{N}$, then the circumcircle of $\mathscr{D} \mathscr{T}$ contains no other nodes of N. In Figure 1(b), the Delaunay circumcircles for three triangles are shown. Consider the introduction of a point $p$ with co-ordinate $\mathbf{x} \in \mathbb{R}^{2}$ into the domain $\Omega$ (Figure $1(\mathrm{~b})$ ). The Voronoi diagram $\mathscr{V}\left(n_{1}, n_{2}, \ldots, n_{M}, p\right)$ or equivalently the Delaunay triangulation $\mathscr{D} \mathscr{T}\left(n_{1}, n_{2}, \ldots, n_{M}, p\right)$ for the $M$ nodes and the point $p$ is constructed. Now, if the Voronoi cell for $p$ and $n_{I}$ have a common facet (segment in $\mathbb{R}^{2}$ and a polygon in $\mathbb{R}^{3}$ ), then the node $n_{I}$ is said to be a natural neighbour of the point $p$ [17]. The Voronoi cells for point $p$ and its natural neighbours are shown in Figure 1(c).

\subsection{Sibson interpolant}

Sibson [17] introduced natural neighbours and natural neighbour (Sibson) interpolation for the purpose of data interpolation and smoothing. The Sibson co-ordinate is based on the firstand second-order Voronoi diagram and is defined by the ratio of area measures in 2D [17]:

$$
\phi_{I}(\mathbf{x})=\frac{A_{I}(\mathbf{x})}{A(\mathbf{x})}, \quad A(\mathbf{x})=\sum_{J=1}^{n} A_{J}(\mathbf{x})
$$

where $A(\mathbf{x})$ is the area of the first-order Voronoi cell of $p$ and $A_{I}(\mathbf{x})$ is the area of overlap between the first-order Voronoi cells of $p$ and node $I$ (Figure 2(a)). If the point $\mathbf{x} \rightarrow \mathbf{x}_{I}$, then $\phi_{I}(\mathbf{x})=1$ and all other shape functions are zero. The properties of positivity, interpolation, 

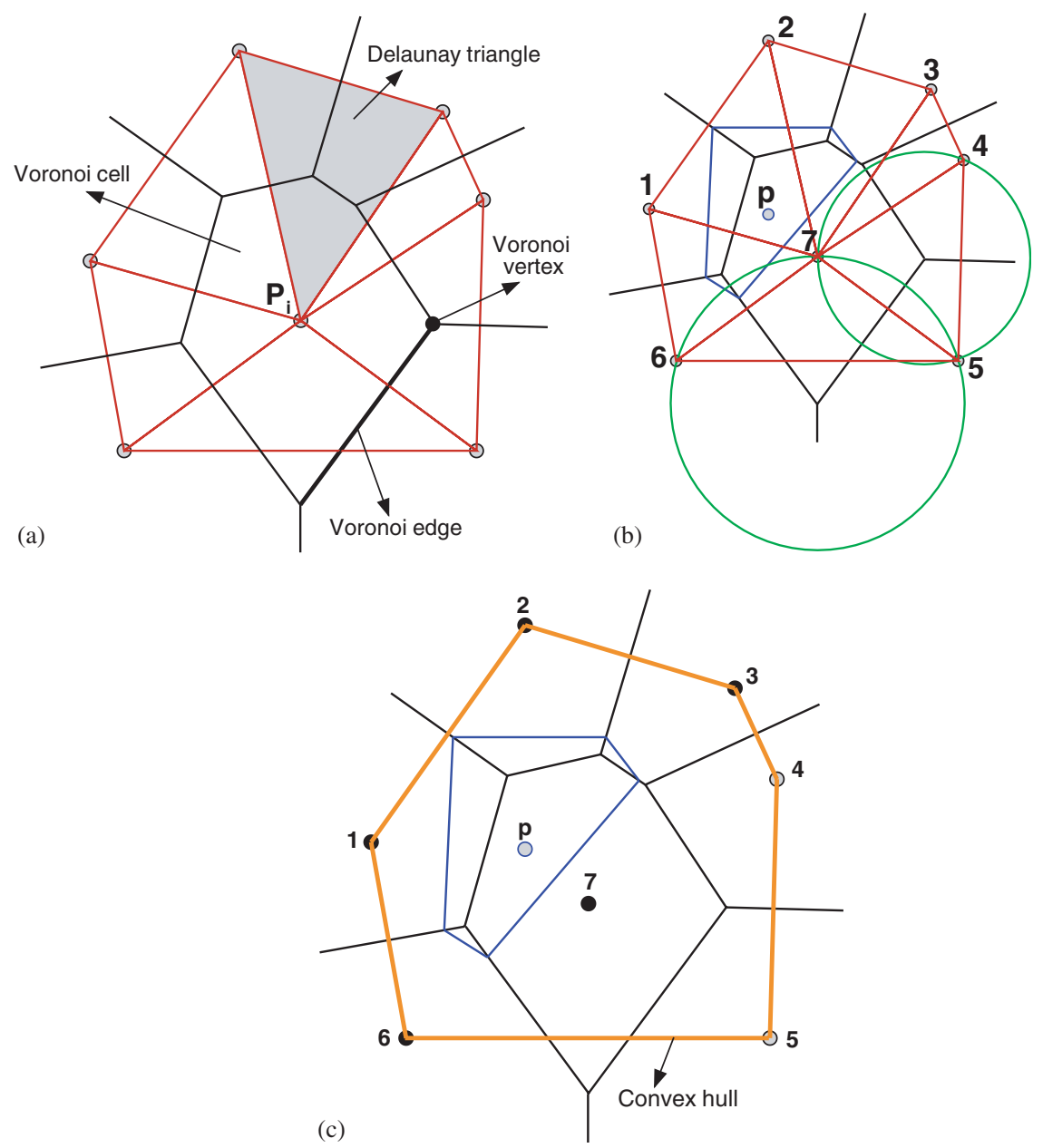

Figure 1. Geometric constructs: (a) Voronoi diagram and Delaunay triangulation; (b) Delaunay circumcircles; and (c) natural neighbours (filled circles) of inserted point $p$.

and partition of unity follow [29]:

$$
0 \leqslant \phi_{I} \leqslant 1, \quad \phi_{I}\left(\mathbf{x}_{J}\right)=\delta_{I J}, \quad \sum_{I=1}^{n} \phi_{I}(\mathbf{x})=1
$$

Natural neighbour shape functions also satisfy the local co-ordinate property [17], namely

$$
\mathbf{x}=\sum_{I=1}^{n} \phi_{I}(\mathbf{x}) \mathbf{x}_{I}
$$

and hence the Sibson interpolant can exactly represent any linear field, which is known as linear completeness in the finite element literature. Further details on the Sibson interpolant and its application to PDEs can be found in Reference [29] and the references therein. 

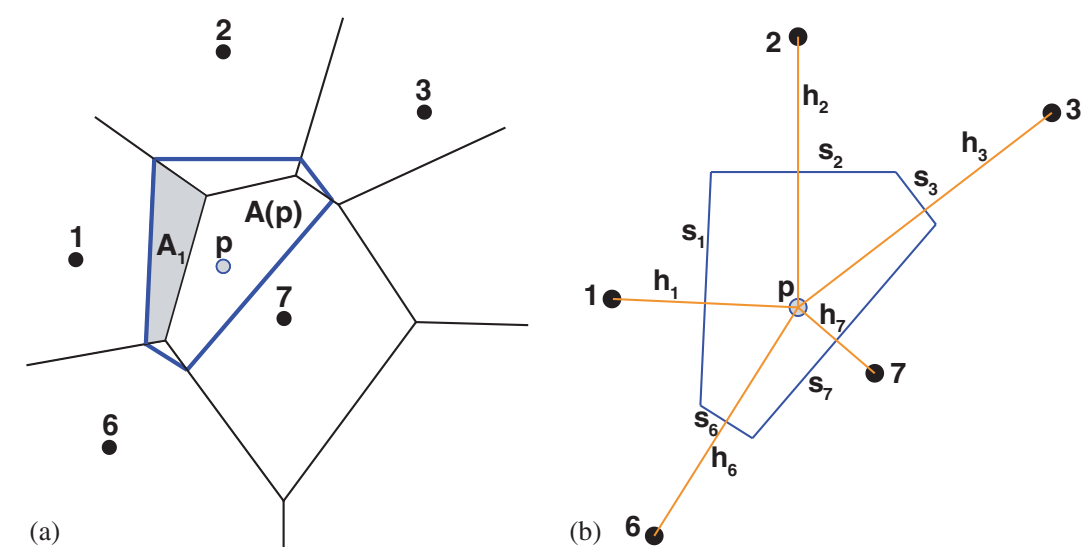

Figure 2. Natural neighbour-based interpolants: (a) Sibson interpolant; and (b) Laplace interpolant.

\subsection{Laplace interpolant}

As first noted in Reference [33], Belikov and co-workers [21-23] as well as Hiyoshi and Sugihara [24-26] independently proposed a natural neighbour-based interpolant that was different from the Sibson interpolant. The former scientists who are in the field of data approximation and PDEs referred to the new interpolant as the non-Sibsonian interpolant, whereas Hiyoshi and Sugihara (computational geometers) coined it as the Laplace interpolant. Both the groups recognized its connection to the Laplace equation, and used very similar approaches in delineating many of its properties. In this paper, we choose to refer to this interpolant as the Laplace interpolant. In References [21-23], tools from vector calculus and data approximation theory are used to investigate the Laplace interpolant; Hiyoshi and Sugihara [24-26] use the Minkowski theorem for convex polytopes [46] (Gauss's divergence theorem) and geometric-based theorems to study the Sibson and the Laplace interpolants. They view the Laplace and Sibson interpolants as particular instances of Voronoi-based interpolants, and present a framework for generating a hierarchy of natural-neighbour based interpolants with increasing order of continuity at non-nodal locations. The Sibson interpolant can be obtained by the Voronoi-based integration of the Laplace interpolant and therein lies a reason why the Sibson interpolant is smoother than the Laplace interpolant [26]. By proceeding further, one can show that a limiting argument in the definition of the Laplace interpolant leads to the three-node Delaunay interpolant (constant strain finite elements). The contributions from both groups bring out differing viewpoints and perspectives in regard to the interpolantproperties, applications, and connections to other data approximation techniques. The link to Galerkin finite elements for both these interpolants is presented in References [27, 29, 32].

The above findings in regard to the Laplace interpolant are, however, preceded by earlier work [42] on the Laplace weight that escaped the attention of the aforementioned researchers. In a series of papers, Christ and co-workers [40-42] investigated the possibility of carrying out quantum field theory computations in a discrete setting. Regular lattices violate some of the fundamental invariance postulates in quantum theory, and hence they considered the replacement of the space-time continuum by a random lattice. A discrete description of the continuum was obtained by using the Delaunay tessellation of all the sites. They 
associated the Laplace weight [42] to the Delaunay edges (link that connects two sites) in the tessellation. In Reference [42], the divergence theorem is used to prove Equation (4) as well as other identities on the lattice that are obeyed by the Laplace weights.

The Laplace shape function is first defined in $d$-dimensions, and then, in keeping with the applications pursued in this paper, we focus our attention on the $2 \mathrm{D}$ case. Let $\mathbf{N}$ denote a nodal set which was defined in Section 2, with the associated Voronoi cell for node $I$ given in Equation (1). Let $t_{I J}$ be the $(d-1)$-dimensional facet (segment in $2 \mathrm{D}$ and polygon in 3D) that is common to $V_{I}$ and $V_{J}$, and $m\left(t_{I J}\right)$ denote the Lebesgue measure of $t_{I J}$, i.e., a length in $2 \mathrm{D}$ and an area in 3D. If $I$ and $J$ do not have common facet, then $m\left(t_{I J}\right)=0$. Now, consider the introduction of a point $p$ with co-ordinate $\mathbf{x} \in \mathbb{R}^{d}$ into the tessellation. If the point $p$ has $n$ natural neighbours, then the Laplace shape function for node $I$ is defined as [21,42]:

$$
\phi_{I}(\mathbf{x})=\frac{\alpha_{I}(\mathbf{x})}{\sum_{J=1}^{n} \alpha_{J}(\mathbf{x})}, \quad \alpha_{J}(\mathbf{x})=\frac{m\left(t_{J}(\mathbf{x})\right)}{h_{J}(\mathbf{x})}, \quad \mathbf{x} \in \mathbb{R}^{d}
$$

In $2 \mathrm{D}$, the above equation takes the form

$$
\phi_{I}(\mathbf{x})=\frac{\alpha_{I}(\mathbf{x})}{\sum_{J=1}^{n} \alpha_{J}(\mathbf{x})}, \quad \alpha_{J}(\mathbf{x})=\frac{s_{J}(\mathbf{x})}{h_{J}(\mathbf{x})}, \quad \mathbf{x} \in \mathbb{R}^{2}
$$

where $\alpha_{J}(\mathbf{x})$ is the Laplace weight function, $s_{I}(\mathbf{x})$ is the length of the Voronoi edge associated with $p$ and node $I$, and $h_{I}(\mathbf{x})$ is the Euclidean distance between $p$ and node $I$ (Figure 2(b)). In $2 \mathrm{D}$, the Laplace shape function involves ratio of length measures whereas the Sibson shape function (see Equation (2)) is based on the ratio of areas. Hence, the computational costs favour the Laplace interpolant in $2 \mathrm{D}$, with the advantage becoming even more significant in $3 \mathrm{D}$.

Let $\mathbf{x} \in \Omega$ be a point in the plane. The Laplace interpolation scheme for a scalar-valued function $u(\mathbf{x}): \Omega \rightarrow \mathbb{R}$ is written as

$$
u^{h}(\mathbf{x})=\sum_{I=1}^{n} \phi_{I}(\mathbf{x}) u_{I}
$$

where $u_{I}(I=1,2, \ldots, n)$ are the unknowns at the $n$ natural neighbours, and $\phi_{I}(\mathbf{x})$ is the Laplace shape function associated with node $I$. In Reference [32], the Laplace interpolant is used as trial and test functions in a Galerkin method for applications in 2D elasticity.

2.2.1. Properties. The Laplace shape functions are strictly positive, interpolate nodal data and also form a partition of unity (see Equation (3)). The domain of support (region in which $\left.\phi_{I}>0\right)$ of the Laplace shape function $\phi_{I}$ is the union of Delaunay circumcircles about node $I$ (Figure 3), and they also form a linearly complete approximation [42]. In these respects, the Laplace and the Sibson shape functions share the same properties. For a domain $\Omega$ with boundary $\partial \Omega$, we can write

$$
\int_{\Omega} \nabla f \mathrm{~d} \Omega=\int_{\partial \Omega} f \mathbf{n} \mathrm{d} \Gamma
$$

by virtue of Gauss's theorem. On setting $f=1$, we have

$$
\int_{\partial \Omega} \mathbf{n} \mathrm{d} \Gamma=\mathbf{0}
$$




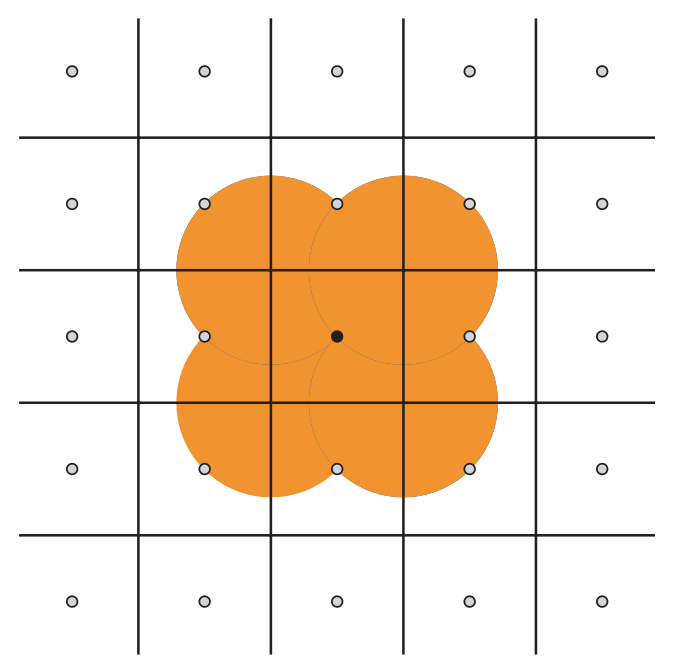

Figure 3. Support of natural neighbour-based shape functions.

which is also known as the Minkowski theorem [46] for convex polytopes in computational geometry. Now, on discretizing the above integral over the Voronoi cell $\left(\Omega \equiv A_{p}\right)$ of point $p$ with co-ordinate $\mathbf{x}$, we obtain $[24,42]$ :

$$
\sum_{I=1}^{n} \frac{\mathbf{x}_{I}-\mathbf{x}}{h_{I}(\mathbf{x})} s_{I}(\mathbf{x})=\mathbf{0}
$$

and therefore

$$
\mathbf{x}=\sum_{I=1}^{n} \phi_{I}(\mathbf{x}) \mathbf{x}_{I}, \quad \alpha_{I}(\mathbf{x})=\frac{s_{I}(\mathbf{x})}{h_{I}(\mathbf{x})}, \quad \phi_{I}(\mathbf{x})=\frac{\alpha_{I}(\mathbf{x})}{\sum_{J} \alpha_{J}(\mathbf{x})}
$$

which is the linear reproducing condition in Equation (4).

The distinction of the Sibson and the Laplace shape functions is notable in two key areas: smoothness and symmetry. The Sibson shape function is $C^{1} \backslash \mathbf{x}_{I}\left(C^{0}\right.$ at nodal locations) [18], whereas the Laplace shape function is $C^{0}$ at nodal locations as well as on the boundary of the support (see Figure 3) [26]. The Laplace weight function is symmetric $\left(\alpha_{I J}=\alpha_{J I}\right)$, but the Sibson weight is not. This property of the Laplace weight $\alpha_{I J}$ proves to be of particular importance in the construction of the Voronoi cell finite difference scheme that is presented in this paper.

\subsection{Computational algorithm}

A local implementation of the Bowyer-Watson algorithm $[47,48]$ can be readily adopted to compute the Sibson and Laplace interpolants within a Galerkin framework or even within the proposed finite difference scheme as a post-processing tool at non-nodal locations. The sequence of illustrations in Figure 4 indicate the key steps in the evaluation of these interpolants. Given a point $p$, the Delaunay circumcircles that violate the circumcircle criterion ( $p$ lies inside the circle) are found and the internal edges of the associated triangles are deleted (Figures 4(a) and 4(b)). Point $p$ is connected to the facets on the outer boundary 
(a)

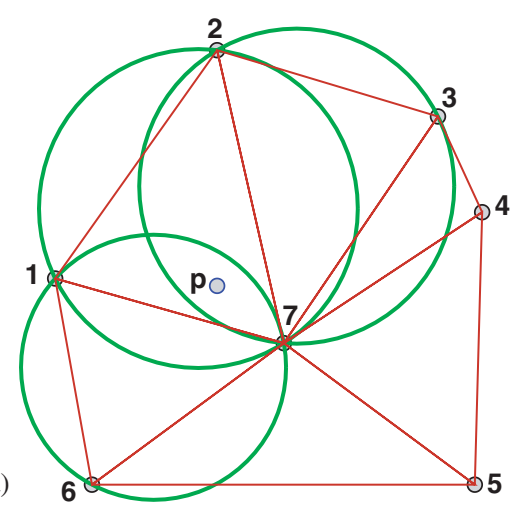

(c)

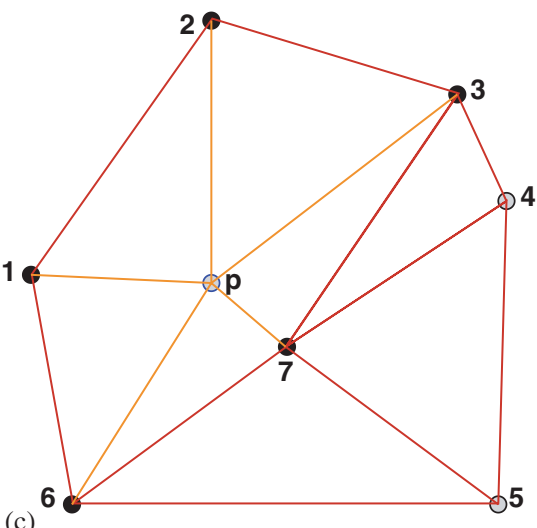

(b)
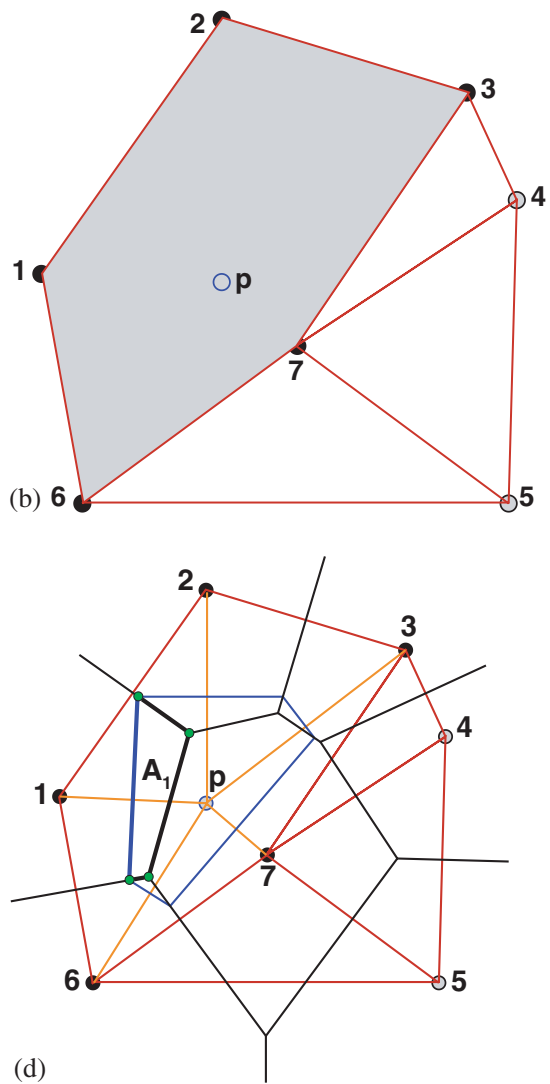

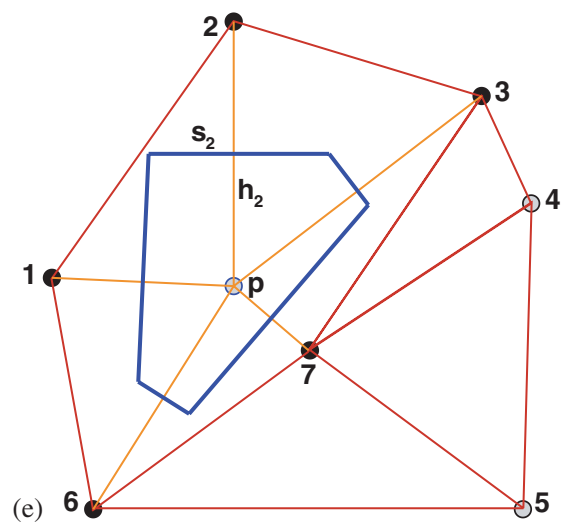

Figure 4. Computational algorithm for natural neighbour-based interpolants: (a) Delaunay triangulation and Delaunay circumcircles; (b) deleted interior facets (edges) to form interior cavity; (c) join boundary facets to point $p$ to form new triangulation; (d) Sibson interpolant defined by overlapping areas of original Voronoi diagram and the Voronoi cell of $p$; and (e) Laplace interpolant defined solely using the Voronoi cell of $p$. 
which defines the new triangulation (Figure 4(c)). Now, using simple geometric computations, the Sibson (Figure 4(d)) and the Laplace shape functions (Figure 4(e)) are evaluated. For the Laplace interpolant which is considered in this paper, the Voronoi edge length $\left(s_{I}\right)$ is computed by computing the distance between the adjacent Voronoi vertices. Simple algebraic formulas (see Reference [29]) for the circumcenter of a triangle are used to evaluate the co-ordinate of the Voronoi vertices. For a triangle $t(A, B, C)$ with vertices $A(\mathbf{a}), B(\mathbf{b})$, and $C(\mathbf{c})$, the circumcenter $\left(v_{1}, v_{2}\right)$ of $t$ is

$$
\begin{aligned}
& v_{1}=\frac{\left(a_{1}^{2}-c_{1}^{2}+a_{2}^{2}-c_{2}^{2}\right)\left(b_{2}-c_{2}\right)-\left(b_{1}^{2}-c_{1}^{2}+b_{2}^{2}-c_{2}^{2}\right)\left(a_{2}-c_{2}\right)}{D} \\
& v_{2}=\frac{\left(b_{1}^{2}-c_{1}^{2}+b_{2}^{2}-c_{2}^{2}\right)\left(a_{1}-c_{1}\right)-\left(a_{1}^{2}-c_{1}^{2}+a_{2}^{2}-c_{2}^{2}\right)\left(b_{1}-c_{1}\right)}{D}
\end{aligned}
$$

where $D$ which is four times the area of triangle $t(A, B, C)$ is given by

$$
D=2\left[\left(a_{1}-c_{1}\right)\left(b_{2}-c_{2}\right)-\left(b_{1}-c_{1}\right)\left(a_{2}-c_{2}\right)\right]
$$

In the above equations, $\mathbf{a}=\left(a_{1}, a_{2}\right), \mathbf{b}=\left(b_{1}, b_{2}\right)$, and $\mathbf{c}=\left(c_{1}, c_{2}\right)$ (counter-clockwise orientation) are the co-ordinates of the vertices of $t$. Further details on the Laplace algorithm are provided in Reference [32].

\section{CONNECTIONS AND APPLICATIONS OF THE LAPLACE INTERPOLANT}

The connections of the Sibson and Laplace interpolants to various numerical techniques and applications are discussed. In previous studies [27-29,32], the Sibson and the Laplace interpolants were shown to reduce to finite element interpolation for certain specific cases: in 1D, these interpolants are the same as linear finite element interpolation and in $2 \mathrm{D}$, if a point $p$ has three neighbours, barycentric co-ordinates are obtained and for the case of four natural neighbours $(n=4)$ at the vertices of a rectangle, bilinear interpolation on the rectangle is obtained [29, 32]. In Section 3.1, the Laplace weight is used to construct a Voronoi cell finite difference scheme that encompasses the classical finite difference scheme as a special case when regular nodal discretizations are considered.

In quantum field theory, the replacement of the space-time continuum by a random lattice with an appropriate weight measure was explored in Reference [42], and the properties of the Laplace weight were studied towards that goal. Lattice and spring-network models for fracture have received a lot of attention [49-51], but previous studies have indicated the inherent difficulties associated with carrying out elastically homogeneous and grid-insensitive fracture simulations on random lattice networks. A partial resolution to the above shortcomings was met in References [52,53], where the Laplace weight is used to successfully perform grid-insensitive crack propagation simulations on Voronoi grids. In References [54], the convergence properties of the non-symmetric random walk are studied using the Laplace weight. These independent applications of the Laplace weight point to the possibility of carrying out numerical computations of continuum field equations on a lattice network. 


\subsection{Voronoi cell finite difference scheme on irregular grids}

The Voronoi tessellation and its dual the Delaunay triangulation are used to discretize a continuum. The Voronoi cell provides a natural domain of influence for a given node, and hence it is commonly used in numerical methods such as the finite volume and the finite element method. In the Voronoi cell finite element method (VCFEM) [55,56], the Voronoi tessellation is used to represent the material microstructure and a finite element formulation is developed on the Voronoi cells. The numerical method is used for multiscale analysis of heterogeneous materials.

Classical finite difference schemes are typically based on regular grids, and hence in most instances, they are used for the numerical solution of PDEs on rectangular domains. The development of finite difference stencils on unstructured grids is an active area of research. Typically, in most of the schemes proposed thus far, a star-shaped domain or computational cell is associated with a node at which the difference approximation is computed. A minimum number of neighbouring nodes are required to be within the computational cell to construct a linear or quadratic finite difference approximation. A system of linear (possibly over-determined) equations is solved at each node to obtain the discrete differential operators $[3,4,6]$.

Our approach assumes a different viewpoint. The Voronoi cell is adopted as the computational cell and an integral balance law is used to derive the finite difference scheme for the diffusion operator. The motivation for the above is derived from prior work on the Laplace interpolant [22,32] and from [57], in which prescriptions are presented for vector identities (such as the gradient and divergence) on a lattice. The approach we pursue bears close connection to that pursued within the context of a finite volume scheme [8], as well as in integrated finite differences [58] which is extensively used in hydrogeology. The finite difference scheme proposed herein can be viewed as a generalization of the approach in Reference [58] to Voronoi grids.

In this paper, we consider the following two-dimensional steady-state diffusion equation with Dirichlet boundary conditions:

$$
\begin{aligned}
-\mathscr{L} u(\mathbf{x}) & =-\nabla \cdot(\kappa(\mathbf{x}) \nabla u(\mathbf{x}))=f(\mathbf{x}) \quad \text { in } \Omega \subset \mathbb{R}^{2} \\
u(\mathbf{x}) & =g(\mathbf{x}) \quad \text { on } \partial \Omega
\end{aligned}
$$

where $\nabla$ is the gradient operator, $\Omega$ is an open set in $2 \mathrm{D}$, and $\partial \Omega$ is the boundary of $\Omega$. If $\kappa \equiv 1$, then $\mathscr{L}$ is the Laplacian operator. The above diffusion equation, which is written in flux form, is used to describe diffusion processes such as heat conduction, mass transfer, flow through porous media, or the potential in electrostatics. In the case of heat conduction, $u$ is the temperature, $\kappa(\mathbf{x})>0$ is the heat conductivity (isotropic heat conduction is assumed), and $f$ is the heat source or sink. The function $g(\mathbf{x})$ is the specified boundary temperature (Dirichlet boundary condition) on $\partial \Omega$.

3.1.1. Finite difference scheme. The model diffusion problem in Equation (13) is solved using a finite difference method, or equivalently a point collocation scheme [59]. The discrete form for the model problem in Equation (13) is written as

$$
-\mathscr{L}_{h} u\left(\mathbf{x}_{I}\right)=f\left(\mathbf{x}_{I}\right), \quad I=1,2, \ldots, M\left(\mathbf{x}_{I} \in \Omega\right)
$$




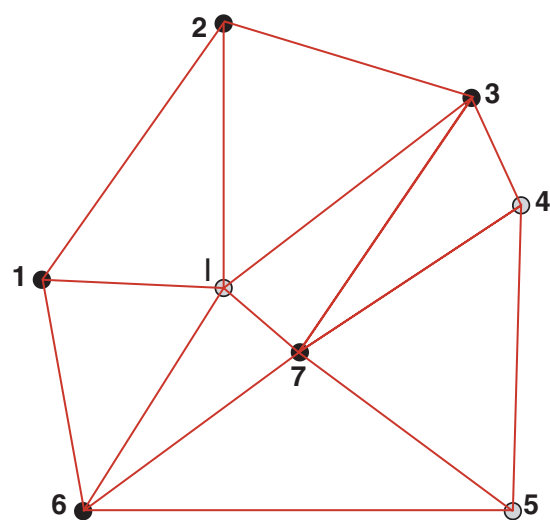

(a)

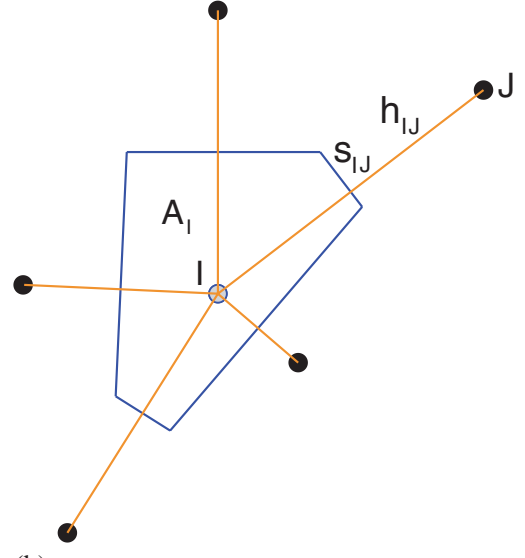

(b)

Figure 5. Finite difference approximation at node $I$ : (a) node $I$ in the triangulation; and (b) Voronoi cell of node $I$ and its natural neighbours.

$$
u\left(\mathbf{x}_{I}\right)=g\left(\mathbf{x}_{I}\right) \quad \mathbf{x}_{I} \in \partial \Omega
$$

where $M$ is the number of the nodes in the domain, $\mathscr{L}_{h}$ is the discrete diffusion operator, and $h$ denotes a measure of the nodal spacing.

Let us consider the domain $\Omega$ shown in Figure 1(c) which is reproduced in Figure 5(a). The point $p$ that was added to the tessellation is now assumed to denote a node (say $I$ ). In the exposition presented in the preceding sections, the Sibson and Laplace interpolants are defined for all points $p$ with co-ordinate $\mathbf{x}$ that are contained within the domain. In moving to a discrete setting, we can reconstruct the same picture by imagining that a node (say $I$ that is located at $\mathbf{x}_{I} \equiv \mathbf{x}$ ) has been removed and is then inserted into the grid. Clearly, depending on the context, one can see (conceptually) the equivalence between defining the approximation at a point $p$ (continuum perspective) vis-à-vis that at a node (discrete/lattice perspective) for a given nodal discretization. Proceeding likewise, one can approximate the discrete nodal gradient in $2 \mathrm{D}$ as

$$
(\nabla u)_{I}=\frac{\partial u^{h}}{\partial x_{1}} \mathbf{e}_{1}+\frac{\partial u^{h}}{\partial x_{2}} \mathbf{e}_{2}=\left(\sum_{J=1}^{n_{I}} w_{I J}^{1} u_{J}\right) \mathbf{e}_{1}+\left(\sum_{J=1}^{n_{I}} w_{I J}^{2} u_{J}\right) \mathbf{e}_{2}
$$

where the weight $w_{I J}^{j}\left(\mathbf{x}_{I}\right)$ is the derivative of the Laplace shape function in the $x_{j}$-co-ordinate direction. Other forms for approximating the nodal displacement gradients (strains) have been recently explored $[32,60]$. Chen and co-workers [60] proposed a novel strain smoothing (finite volume averaging) procedure to construct the discrete strain operator in meshless Galerkin methods.

We now proceed to find the discrete approximation for the diffusion operator at node $I$, i.e., $\mathscr{L}_{h} u\left(\mathbf{x}_{I}\right)$ given in Equation (14a). The starting point is the balance (conservation) law for the divergence of the flux over the Voronoi cell $A_{I}$ (see Figure 5(b)). We can write 
(flux form)

$$
(\nabla \cdot \mathbf{q})_{I}=\lim _{A_{I} \rightarrow 0} \frac{\int_{A_{I}} \boldsymbol{\nabla} \cdot \mathbf{q} \mathrm{d} \Omega}{\int_{A_{I}} \mathrm{~d} \Omega}=\lim _{A_{I} \rightarrow 0} \frac{\int_{\partial A_{I}} \mathbf{q} \cdot \mathbf{n} \mathrm{d} \Gamma}{A_{I}}
$$

where Gauss's (divergence) theorem has been invoked, and $A_{I}$ is the area of the Voronoi cell of node $I$. Now, the flux $\mathbf{q}=-\kappa \nabla u$, and hence

$$
[-\nabla \cdot(\kappa(\mathbf{x}) \nabla u)]_{I}=-\lim _{A_{I} \rightarrow 0} \frac{\int_{\partial A_{I}} \kappa(\mathbf{x}) \nabla u \cdot \mathbf{n} \mathrm{d} \Gamma}{A_{I}}=-\lim _{A_{I} \rightarrow 0} \frac{\int_{\partial A_{I}} \kappa(\mathbf{x})(\partial u / \partial n) \mathrm{d} \Gamma}{A_{I}}
$$

To find the discrete form for the diffusion operator we consider the evaluation of the above integral on the boundary of the Voronoi cell of node $I$, and use a simple central-difference (cell-based) approximation for the derivative of $u$ normal to the Voronoi edge (Figure 5(b)). In addition, the diffusive coefficient $\kappa(\mathbf{x})$ is approximated using the harmonic average of its value at the nodes on either side of the Voronoi edge. This choice for $\kappa(\mathbf{x})$ conserves flux across the Voronoi edge in diffusion phenomena such as flow through a heterogeneous medium [58]. Hence, we can now write

$$
\left(\mathscr{L}_{h} u\right)_{I}=\frac{\int_{\partial A_{I}} \kappa(\mathbf{x})\left(\partial u_{h} / \partial n\right) \mathrm{d} \Gamma}{A_{I}}=\frac{1}{A_{I}} \sum_{J=1}^{n} \kappa_{I J} \frac{\left(u_{J}-u_{I}\right)}{h_{I J}} s_{I J}, \quad \frac{2}{\kappa_{I J}}=\frac{1}{\kappa_{I}}+\frac{1}{\kappa_{J}}
$$

where $n$ is the number of natural neighbours for node $I$ ( $n=5$ in Figure $5(\mathrm{~b})), h_{I J}$ is the distance between nodes $I$ and $J$, and $s_{I J}$ is the length of the Voronoi edge associated with nodes $I$ and $J$ (Figure 5(b)). Using Equation (6), the above equation is written as

$$
\begin{aligned}
\left(\mathscr{L}_{h} u\right)_{I} & =\frac{1}{A_{I}}\left[\sum_{J=1}^{n} \kappa_{I J} \alpha_{I J} u_{J}-\left(\sum_{J=1}^{n} \kappa_{I J} \alpha_{I J}\right) u_{I}\right] \\
A_{I} & =\frac{1}{4} \sum_{J=1}^{n} s_{I J} h_{I J}=\frac{1}{4} \sum_{J=1}^{n} \alpha_{I J} h_{I J}^{2}, \quad \alpha_{I J}=\frac{s_{I J}}{h_{I J}}
\end{aligned}
$$

where $\alpha_{I J}$ is the Laplace weight and the Voronoi polygon is decomposed into $n$ triangles with common vertex at $\mathbf{x}_{I}$ to find its area (this approach extends to $3 \mathrm{D}$ too). Denoting $\beta_{I J}=\kappa_{I J} \alpha_{I J}$, we can write

$$
\left(\mathscr{L}_{h} u\right)_{I}=\frac{1}{A_{I}}\left[\sum_{J=1}^{n} \beta_{I J} u_{J}-\beta_{I} u_{I}\right], \quad \beta_{I}=\sum_{J=1}^{n} \beta_{I J}
$$

The above expression is consistent with the prescription introduced for the discrete Laplacian $(\kappa \equiv 1)$ on a random lattice [57]. ${ }^{\ddagger}$ The right-hand side of Equation (14) is just $f_{I}$. Hence,

\footnotetext{
${ }^{\ddagger}$ Note added in the proof: The author recently came across the work of Börgers and Peskin [61, 62], in which an in-depth study on the properties of the discrete Laplacian on Voronoi grids is carried out. In Reference $[61,62]$, geometric computations are also used to show the equivalence of the finite-difference stiffness matrix to that obtained in finite elements. The contribution herein provides a different perspective-emergence of the Laplace weight function in the difference approximation and its use to study the convergence properties of the method.
} 


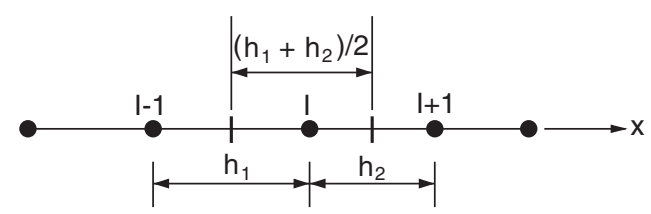

Figure 6. Non-uniform grid in one-dimension.

the discrete system for the finite difference (or collocation) scheme is

$$
\begin{aligned}
\mathbf{K u} & =\tilde{\mathbf{f}} \\
\mathbf{K}_{I I} & =\beta_{I}, \quad \mathbf{K}_{I J}=-\beta_{I J} \quad(I \neq J), \quad \tilde{\mathbf{f}}_{I}=f_{I} A_{I}, \quad\left(\mathbf{x}_{I} \in \Omega\right) \\
u_{I} & =g\left(\mathbf{x}_{I}\right), \quad \mathbf{x}_{I} \in \partial \Omega \\
\beta_{I J} & =\kappa_{I J} \alpha_{I J}, \quad \beta_{I}=\sum_{J=1}^{n} \beta_{I J}, \quad \kappa_{I J}=\frac{2 \kappa_{I} \kappa_{J}}{\kappa_{I}+\kappa_{J}}
\end{aligned}
$$

If $\kappa \equiv 1$, we obtain the Poisson equation. Using

$$
\alpha_{I J}=\frac{s_{I J}}{h_{I J}}, \quad \alpha_{I}=\sum_{J=1}^{n} \alpha_{I J}
$$

the discrete system can now be written as

$$
\mathbf{K}_{I I}=\alpha_{I}, \quad \mathbf{K}_{I J}=-\alpha_{I J} \quad(I \neq J), \quad \tilde{\mathbf{f}}_{I}=f_{I} A_{I}, \quad\left(\mathbf{x}_{I} \in \Omega\right), \quad u_{I}=g\left(\mathbf{x}_{I}\right), \quad \mathbf{x}_{I} \in \partial \Omega
$$

Next, the equivalence to cell-centred finite differences for non-uniform grids in one dimension is shown, and then consistency, positive-definiteness, and stability (via the discrete maximum principle) of the proposed finite difference scheme are studied.

3.1.2. Non-uniform grid in one-dimension. Consider the finite difference approximation for the Laplacian $\left(\mathscr{L} u=u_{x x}\right)$ on a one-dimensional non-uniform grid (Figure 6). The discrete Laplacian is computed at node $I$, whose natural neighbours are $I-1$ and $I+1$. The Voronoi cell of node $I$ is shown in Figure 6; the length of the cell is $\ell_{I}=\left(h_{1}+h_{2}\right) / 2$, where $h_{1}=x_{I}-x_{I-1}$ and $h_{2}=x_{I+1}-x_{I}$ are the grid spacings. In $1 \mathrm{D}$, the Voronoi facet reduces to a point and hence the definition for $s_{I J}$ cannot be directly used (the Lebesgue measure of a point is zero). The term $s_{I J}$ arises when the volume integral is transformed to a surface integral (Gauss's theorem). In $1 \mathrm{D}, \int_{x_{1}}^{x_{2}} f_{, x} \mathrm{~d} x=f\left(x_{2}\right)-f\left(x_{1}\right)$ by the fundamental theorem of calculus (unit normal is +1 for $x=x_{2}$ and -1 for $\left.x=x_{1}\right)$ and hence the choice $s_{I-1}=s_{I+1} \equiv 1$ is appropriate. In addition, we have $h_{I-1}=h_{1}$ and $h_{I+1}=h_{2}$. Using Equation (20), the discrete Laplacian $(\kappa \equiv 1)$ for node $I$ can be written as

$$
\left(\mathscr{L}_{h} u\right)_{I}=\frac{1}{\ell_{I}}\left[\sum_{J=1}^{2} \alpha_{I J} u_{J}-\alpha_{I} u_{I}\right], \quad \alpha_{I}=\sum_{J=1}^{2} \alpha_{I J}
$$

which becomes

$$
\left(\mathscr{L}_{h} u\right)_{I}=\frac{2}{h_{1}+h_{2}}\left[\frac{1}{h_{1}} u_{I-1}+\frac{1}{h_{2}} u_{I+1}-\left(\frac{1}{h_{1}}+\frac{1}{h_{2}}\right) u_{I}\right]
$$


or

$$
\left(\mathscr{L}_{h} u\right)_{I}=\frac{2}{h_{1} h_{2}\left(h_{1}+h_{2}\right)}\left(h_{2} u_{I-1}+h_{1} u_{I+1}-\left(h_{1}+h_{2}\right) u_{I}\right)
$$

If $h_{1}=h_{2}=h$ (uniform grid), we obtain the classical central-difference approximation:

$$
\left(\mathscr{L}_{h} u\right)_{I}=\frac{u_{I-1}+u_{I+1}-2 u_{I}}{h^{2}}
$$

and if for example $h_{1}=3 \Delta x / 4, h_{2}=3 \Delta x / 2$, then

$$
\left(\mathscr{L}_{h} u\right)_{I}=\frac{16}{27 \Delta x^{2}}\left(2 u_{I-1}-3 u_{I}+u_{I+1}\right)
$$

which is identical to the expression given in Reference [63], where cell-centred finite difference schemes are derived on non-uniform grids. In Reference [63], Taylor series expansion and the method of undetermined coefficients are used to find the weights so that the coefficient of the first derivative vanishes, coefficient of the second derivative is unity, etc. On using Equation (26), these conditions are automatically satisfied.

3.1.3. Consistency. Consistency in finite difference schemes ensures that in the limit when the grid spacing tends to zero, the difference between the finite difference scheme for the differential operator and the continuous form of the same is zero. We can formally write the following definition for consistency [64]:

\section{Definition}

Given a partial difference equation $\mathscr{L} v=f$ and a finite difference scheme $\mathscr{L}_{h} v=f$, we say that the finite difference scheme is consistent with the partial differential equation if for any smooth function $u$,

$$
\mathscr{L} u-\left(\mathscr{L}_{h} u\right)_{I} \rightarrow 0 \quad \forall I \text { as } h_{I J} \rightarrow 0 \quad(J=1, \ldots, n)
$$

where $h_{I J}$ is the distance between nodes $I$ and $J$ (neighbour), and $n$ is the number of neighbours for node $I$.

We use the Taylor series expansion in terms of directional derivatives to study consistency for the Laplace operator. Consider a direction $n$ with unit vector $\mathbf{n}=\left(n_{x}, n_{y}\right)$ and let $h$ be a scalar. The Taylor series approximation for a function $u(\mathbf{x})$ at $\tilde{\mathbf{x}}=\mathbf{x}+h \mathbf{n}$ is written as

$$
u(\mathbf{x}+h \mathbf{n})=u(\mathbf{x})+\nabla u(\mathbf{x}) \cdot \mathbf{n} h+\left(u_{x x}(\mathbf{x}) n_{x}^{2}+2 u_{x y}(\mathbf{x}) n_{x} n_{y}+u_{y y}(\mathbf{x}) n_{y}^{2}\right) \frac{h^{2}}{2}+\mathcal{O}\left(h^{3}\right)
$$

where $u_{x} \equiv \partial u / \partial x, u_{x x} \equiv \partial^{2} u / \partial x^{2}$, etc. Now, let us consider the finite difference approximation for the Laplacian operator at node $I$ (see Figure 5(b)). Using Equation (30), the Taylor series expansion at a neighbour (node $J$ ) is

$$
u_{J}=u_{I}+\nabla u \cdot \mathbf{n}^{I J} h_{I J}+\left(u_{x x}\left(n_{x}^{I J}\right)^{2}+2 u_{x y} n_{x}^{I J} n_{y}^{I J}+u_{y y}\left(n_{y}^{I J}\right)^{2}\right) \frac{h^{2}}{2}+\mathcal{O}\left(h_{I J}^{3}\right)
$$

where all the derivatives are evaluated at $\mathbf{x}_{I}$, and $\mathbf{n}^{I J}=\left(n_{x}^{I J}, n_{y}^{I J}\right)$ is the unit normal to the Voronoi edge (see Figure 5(b)). On multiplying both sides of the above equation by $s_{I J} / h_{I J}$ 
( $s_{I J}$ is the length of the Voronoi edge) and summing over all the neighbours $(J=1 \rightarrow n)$, we obtain

$$
\begin{aligned}
\sum_{J=1}^{n} \frac{s_{I J}}{h_{I J}} u_{J}= & \sum_{J=1}^{n} \frac{s_{I J}}{h_{I J}} u_{I}+\nabla u \cdot \sum_{J=1}^{n} \frac{s_{I J}}{h_{I J}} \mathbf{n}^{I J} h_{I J} \\
& +\sum_{J=1}^{n} \frac{s_{I J}}{h_{I J}}\left(u_{x x}\left(n_{x}^{I J}\right)^{2}+2 u_{x y} n_{x}^{I J} n_{y}^{I J}+u_{y y}\left(n_{y}^{I J}\right)^{2}\right) \frac{h_{I J}^{2}}{2}+\mathcal{O}\left(h_{m I J}^{3}\right)
\end{aligned}
$$

where $h_{m I J}=\max _{J} h_{I J}$. Since $\sum_{J} s_{I J} h_{I J}=\mathcal{O}\left(h_{m I J}^{2}\right)$ from Equation (19b), the last term which is of the form $\sum_{J} s_{I J} h_{I J}^{2}$ is $\mathcal{O}\left(h_{m I J}^{3}\right)$ after the summation is carried out. The second term on the right-hand side can be simplified as follows:

$$
\nabla u \cdot \sum_{J=1}^{n} s_{I J} \mathbf{n}^{I J}=\nabla u \cdot \sum_{J=1}^{n} s_{I J} \frac{\mathbf{x}_{J}-\mathbf{x}_{I}}{h_{I J}}
$$

and hence on using Equation (10), we have

$$
\nabla u \cdot \sum_{J=1}^{n} s_{I J} \mathbf{n}^{I J}=0
$$

On using the above result and the definitions for $\alpha_{I J}$ and $\alpha_{I}$ that appear in Equation (22), Equation (32) simplifies to

$$
\sum_{J=1}^{n} \alpha_{I J} u_{J}-\alpha_{I} u_{I}=\sum_{J=1}^{n} \alpha_{I J}\left(u_{x x}\left(n_{x}^{I J}\right)^{2}+2 u_{x y} n_{x}^{I J} n_{y}^{I J}+u_{y y}\left(n_{y}^{I J}\right)^{2}\right) \frac{h_{I J}^{2}}{2}+\mathcal{O}\left(h_{m I J}^{3}\right)
$$

Writing out the normal components (for e.g., $n_{x}^{I J}=\left(x_{J}-x_{I}\right) / h$ ), we have

$$
\sum_{J=1}^{n} \alpha_{I J} u_{J}-\alpha_{I} u_{I}=\sum_{J=1}^{n} \frac{\alpha_{I J}}{2}\left(u_{x x}\left(x_{J}-x_{I}\right)^{2}+2 u_{x y}\left(x_{J}-x_{I}\right)\left(y_{J}-y_{I}\right)+u_{y y}\left(y_{J}-y_{I}\right)^{2}\right)+\mathcal{O}\left(h_{m I J}^{3}\right)
$$

Let

$$
a_{I}=\sum_{J=1}^{n} \frac{\alpha_{I J}}{2}\left(x_{J}-x_{I}\right)^{2}, \quad b_{I}=\sum_{J=1}^{n} \frac{\alpha_{I J}}{2}\left(y_{J}-y_{I}\right)^{2}, \quad \zeta_{I}=\sum_{J=1}^{n} \alpha_{I J}\left(x_{J}-x_{I}\right)\left(y_{J}-y_{I}\right)
$$

Then, Equation (36) can be written as

$$
\sum_{J=1}^{n} \alpha_{I J} u_{J}-\alpha_{I} u_{I}=u_{x x} a_{I}+u_{y y} b_{I}+u_{x y} \zeta_{I}+\mathcal{O}\left(h_{m I J}^{3}\right)
$$

Using $a_{I}$ and $b_{I}$, we can write the following identities:

$$
a_{I}=\frac{a_{I}+b_{I}}{2}+\frac{a_{I}-b_{I}}{2}, \quad b_{I}=\frac{a_{I}+b_{I}}{2}-\frac{a_{I}-b_{I}}{2}
$$

or

$$
a_{I}=\sum_{J=1}^{n} \frac{\alpha_{I J} h_{I J}^{2}}{4}+\eta_{I}=A_{I}+\eta_{I}, \quad b_{I}=\sum_{J=1}^{n} \frac{\alpha_{I J} h_{I J}^{2}}{4}-\eta_{I}=A_{I}-\eta_{I}
$$


where $A_{I}$ is the area of the Voronoi cell of node $I$ and $\eta_{I}$ is given by

$$
\eta_{I}=\sum_{J=1}^{n} \frac{\alpha_{I J}}{4}\left(\left(x_{J}-x_{I}\right)^{2}-\left(y_{J}-y_{I}\right)^{2}\right)
$$

Using the above relations, Equation (38) becomes

$$
\sum_{J=1}^{n} \alpha_{I J} u_{J}-\alpha_{I} u_{I}=u_{x x}\left(A_{I}+\eta_{I}\right)+u_{y y}\left(A_{I}-\eta_{I}\right)+u_{x y} \zeta_{I}+\mathcal{O}\left(h_{m I J}^{3}\right)
$$

or

$$
\sum_{J=1}^{n} \alpha_{I J} u_{J}-\alpha_{I} u_{I}=\nabla^{2} u A_{I}+\left(u_{x x}-u_{y y}\right) \eta_{I}+u_{x y} \zeta_{I}+\mathcal{O}\left(h_{m I J}^{3}\right)
$$

We now develop estimates for $\eta_{I}$ and $\zeta_{I}$. For each $I-J$ pair, we can write the following relations:

$$
x_{J}-x_{I}=h_{I J} \cos \theta_{I J}, \quad y_{J}-y_{I}=h_{I J} \sin \theta_{I J}
$$

and hence $\eta_{I}$ in Equation (40b) and $\zeta_{I}$ in Equation (37) can be expressed as

$$
\begin{aligned}
& \eta_{I}=\frac{1}{4} \sum_{J=1}^{n} \alpha_{I J} h_{I J}^{2}\left(\cos ^{2} \theta_{I J}-\sin ^{2} \theta_{I J}\right)=\frac{1}{4} \sum_{J=1}^{n} \alpha_{I J} h_{I J}^{2} \cos 2 \theta_{I J}<\sum_{J=1}^{n} \frac{\alpha_{I J} h_{I J}^{2}}{4} \\
& \zeta_{I}=\sum_{J=1}^{n} \alpha_{I J} h_{I J}^{2} \cos \theta_{I J} \sin \theta_{I J}=\frac{1}{2} \sum_{J=1}^{n} \alpha_{I J} h_{I J}^{2} \sin 2 \theta_{I J}<2 \sum_{J=1}^{n} \frac{\alpha_{I J} h_{I J}^{2}}{4}
\end{aligned}
$$

and therefore

$$
\eta_{I}<A_{I} \Rightarrow \eta_{I}=\mathcal{O}\left(h_{m I J}^{2}\right), \quad \zeta_{I}<2 A_{I} \Rightarrow \zeta_{I}=\mathcal{O}\left(h_{m I J}^{2}\right)
$$

are the best estimates that can be inferred from the above inequality; sharper estimates such as $\mathcal{O}\left(h_{m I J}^{2+\delta}\right)(\delta>0)$ cannot be established.

From Equation (20), we can write the finite difference approximation for the Laplacian as

$$
\mathscr{L}_{h} u=\frac{1}{A_{I}}\left[\sum_{J=1}^{n} \alpha_{I J} u_{J}-\alpha_{I} u_{I}\right]
$$

and on using Equation (45) and the above equation, we can re-write Equation (42) as

$$
\mathscr{L}_{h} u=\nabla^{2} u+\mathcal{O}(1)+\mathcal{O}\left(h_{m I J}\right)
$$

since $A_{I}=\mathcal{O}\left(h_{m I J}^{2}\right)$, and therefore in the limit that the grid spacing tends to zero, we have

$$
\lim _{h_{m I J} \rightarrow 0} \mathscr{L} u-\mathscr{L}_{h} u=-\mathcal{O}(1)
$$

and hence only zeroth-order consistency is obtained. Truncation error norm computations for the Poisson problems considered in Section 4.1.2 are in agreement with the above estimate.

The above result, however, is not surprising since as opposed to uniform grids in one and two dimensions, the satisfaction of consistency on irregular grids is in general difficult. However, the lack of consistency on non-uniform grids as inferred from a Taylor series expansion does not preclude convergence [65-68]. This clearly shows that the treatment of consistency 
and convergence for finite difference schemes on irregular grids is distinctly different from that on uniform grids where consistency (via a Taylor series expansion) and stability are necessary and sufficient conditions for convergence (Lax-Richtmyer theorem) [64].

Kreiss et al. [66] coined the term supraconvergence for schemes that converge at a higher order than the local truncation error; supraconvergence has received a lot of attention in the numerical analysis literature for vertex-and cell-centred finite difference schemes. Lazarov et al. [69] provided a theoretical framework to analyse finite volume cell-centred schemes on uniform Cartesian product grids, whereas in Reference [68], the convergence of block-centred schemes on non-uniform grids is studied. Jones and Menzies [10] showed that cell-centred Cartesian product non-uniform grids are inconsistent via a Taylor series expansion, and extended Süli's analysis [7] to show that the scheme was however convergent. As opposed to finite difference schemes on regular grids, in finite volume (difference) schemes with irregular nodal spacing, the notion of flux consistency appears to be the key ingredient to prove convergence.

The finite difference scheme in this paper is derived from a local conservation law, satisfies the discrete maximum principle as shown in Section 3.1.5, and passes the patch test for the Laplace operator (Section 4.1.1). Using the finite volume formulation as a starting point, Mishev [8] derived error estimates for a cell-centred difference scheme on Voronoi meshes. Clearly, in the present instance, since the finite difference scheme can also be seen in light of a finite volume (conservation) method [8], the traditional Taylor series expansion technique is not adequate to address consistency, and the convergence analysis conducted in References $[8,10]$ is to be followed. Remarks on the importance of consistency of the numerical flux and the use of a conservation form are also apropos in the development of error estimates for the numerical solution of scalar conservation laws on irregular Cartesian product grids [70]. In this study, we do observe the supraconvergence behaviour with second-order convergence in the $L^{2}$ norm for Poisson problems (Section 4.1.2 and 4.1.3).

We now show that if the grid is regular (rectangular or hexagonal), the finite difference scheme is consistent. To this end, using Gauss's theorem, we can write the following identities:

$$
\begin{aligned}
& \int_{A_{I}} \frac{\partial\left(x-x_{I}\right)}{\partial x} \mathrm{~d} \Omega=\int_{\partial A_{I}}\left(x-x_{I}\right) n_{x} \mathrm{~d} \Gamma=A_{I} \\
& \int_{A_{I}} \frac{\partial\left(y-y_{I}\right)}{\partial y} \mathrm{~d} \Omega=\int_{\partial A_{I}}\left(y-y_{I}\right) n_{y} \mathrm{~d} \Gamma=A_{I} \\
& \int_{A_{I}} \frac{\partial\left(x-x_{I}\right)}{\partial y} \mathrm{~d} \Omega=\int_{\partial A_{I}}\left(x-x_{I}\right) n_{y} \mathrm{~d} \Gamma=0
\end{aligned}
$$

Consider Equation (49a) and discretize it on the boundary of the Voronoi cell. Then, we can write [42]

$$
\sum_{J=1}^{n}\left(x_{I J}^{\mathrm{c}}-x_{I}\right) \frac{x_{J}-x_{I}}{h_{I J}} s_{I J}=A_{I}, \quad x_{I J}^{\mathrm{c}}=\frac{\int_{S_{I J}} x \mathrm{~d} s}{s_{I J}}
$$

where $x_{I J}^{\mathrm{c}}$ is the $x$-co-ordinate of the centroid of mass of the Voronoi edge $s_{I J}$. In general, the location of the centre of mass of the Voronoi facet is not known a priori. If the Delaunay 


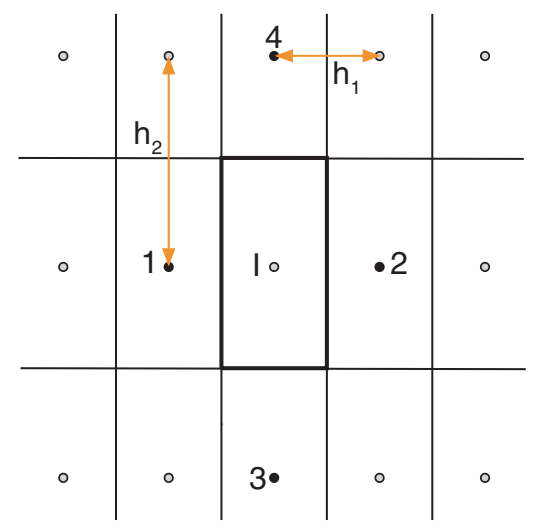

Figure 7. Finite difference approximation for the Laplacian on a rectangular grid with nodal spacings $h_{1}$ and $h_{2}$ in the co-ordinate directions. The Voronoi cell of node $I$ is indicated by the four dark lines that form a rectangle of area $h_{1} h_{2}$.
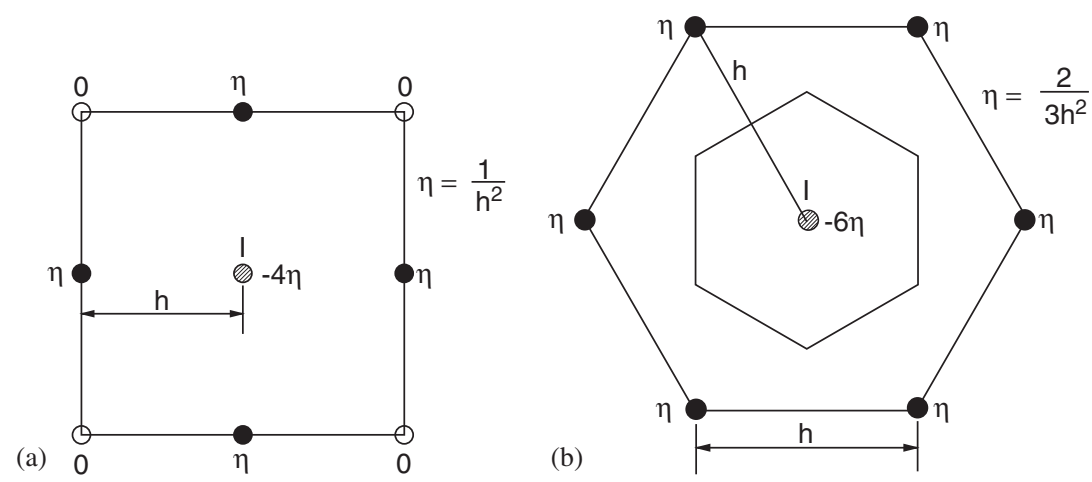

Figure 8. Finite difference weights for the Laplacian on regular grids. Filled circles are the natural neighbours of the interior node and the weights for each neighbour are indicated: (a) square grid; and (b) hexagonal grid.

edge $\left(\mathbf{x}_{I}, \mathbf{x}_{J}\right)$ bisects the Voronoi facet, then $x_{I J}^{\mathrm{c}}=\left(x_{I}+x_{J}\right) / 2$. This is rigorously true for regular grids (see Figures 7 and 8 ), and hence the above equation reduces to

$$
\sum_{J=1}^{n} \frac{\alpha_{I J}}{2}\left(x_{J}-x_{I}\right)^{2}=A_{I}
$$

and proceeding likewise with Equations (49b) and (49c), we obtain

$$
\sum_{J=1}^{n} \frac{\alpha_{I J}}{2}\left(y_{J}-y_{I}\right)^{2}=A_{I}, \quad \sum_{J=1}^{n} \frac{\alpha_{I J}}{2}\left(x_{J}-x_{I}\right)\left(y_{J}-y_{I}\right)=0
$$


Using the above results in Equation (36), with the observation that the coefficient of the $h_{m I J}^{3}$ term also vanishes (neighbour pairs with unit normals $\pm \mathbf{n}$ exist) on a regular grid, we obtain

$$
\sum_{J=1}^{n} \alpha_{I J} u_{J}-\alpha_{I} u_{I}=u_{x x} A_{I}+u_{y y} A_{I}+\mathcal{O}\left(h_{m I J}^{4}\right)
$$

which reduces to

$$
\mathscr{L}_{h} u=\mathscr{L} u+\mathcal{O}\left(h_{m I J}^{2}\right)
$$

since $A_{I}=\mathcal{O}\left(h_{m I J}^{2}\right)$, and therefore

$$
\lim _{h_{m I J} \rightarrow 0} \mathscr{L} u-\mathscr{L}_{h} u=0
$$

and hence the finite difference scheme is consistent on a regular grid.

\section{Remark}

In one dimension, on using the Taylor series expansion, second-order and first-order consistency are established for regular and irregular nodal discretizations, respectively [38].

3.1.4. Positive-definiteness. The invertibility (positive-definiteness) of the discrete matrix $\mathbf{K}$ is studied. To this end, it suffices if we can show that [63]

$$
\begin{gathered}
\left(-\mathscr{L}_{h} v, v\right)>0 \quad \forall v \neq 0 \quad(v=0 \text { on } \partial \Omega) \\
(v, w)=\sum_{I=1}^{M} A_{I} v_{h}\left(\mathbf{x}_{I}\right) w_{h}\left(\mathbf{x}_{I}\right)
\end{gathered}
$$

where $M$ is the number of nodes in the grid, $v$ and $w$ are functions defined on the grid $\left(v\left(\mathbf{x}_{I}\right)=v_{I}, w\left(\mathbf{x}_{I}\right)=w_{I}\right), A_{I}$ is the area of the Voronoi cell of node $I$, and the discrete $L^{2}$ inner product defined in Equation (56b) induces the discrete $L^{2}$ norm given in Equation (78). Using Equation (20), we can write (see Reference [8] too):

$$
\left(-\mathscr{L}_{h} v, w\right)=-\sum_{I=1}^{M} \sum_{\substack{J=1 \\ J \neq I}}^{M} \beta_{I J}\left(v_{J}-v_{I}\right) w_{I}
$$

where the second summation has zero contribution for all $J$ which are not neighbours of node $I\left(s_{I J}=0 \Rightarrow \beta_{I J}=0\right)$. By inter-changing $I$ and $J$ in the above equation and noting that $\beta_{I J}=\beta_{J I}$, we can also write the following equality:

$$
\left(-\mathscr{L}_{h} v, w\right)=-\sum_{J=1}^{M} \sum_{\substack{I=1 \\ I \neq J}}^{M} \beta_{J I}\left(v_{I}-v_{J}\right) w_{J}=\sum_{I=1}^{M} \sum_{\substack{J=1 \\ J \neq I}}^{M} \beta_{I J}\left(v_{J}-v_{I}\right) w_{J}
$$

On taking the average of Equations (57) and (58) and substituting $w=v$, we have

$$
\left(-\mathscr{L}_{h} v, v\right)=\frac{1}{2} \sum_{I=1}^{M} \sum_{\substack{J=1 \\ J \neq I}}^{M} \beta_{I J}\left(v_{J}-v_{I}\right)^{2}
$$

which is positive if $v \neq 0$ and equal to zero if and only if $v \equiv 0$, and hence the positivedefiniteness of the discrete diffusion operator is proven. 
We can also sketch an alternate proof of the above using elements from linear algebra. The symmetry of $\mathbf{K}$ is evident from Equation (21). Let $K_{I J}$ denote the $I J$ th entry in the stiffness matrix and $\rho_{I}=\sum_{\substack{J \\ J \neq I}}^{M}\left|K_{I J}\right|$. Then, we have [71]:

\section{Proposition}

If $\mathbf{K}$ is an irreducible diagonally dominant matrix for which $\left|K_{J J}\right|>\rho_{J}$ for at least one $J$, then $\mathbf{K}$ is invertible. The matrix $\mathbf{K}$ is said to be diagonally dominant if $\left|K_{J J}\right| \geqslant \rho_{J}(J=1,2, \ldots, M)$.

The matrix $\mathbf{K}$ for the diffusion operator in Equation (21) is irreducible (as are most matrices obtained from finite difference equations [71]). In addition, from Equation (21), we have

$$
\left|K_{I I}\right|=\beta_{I}, \quad \rho_{I}=\sum_{K=1}^{n} \beta_{I K}=\beta_{I}
$$

where $n$ is the number of natural neighbours of node $I$. From the above, we note that the diagonal entry in any row is equal to the sum of the magnitude of the off-diagonal entries on the same row. Hence, $\mathbf{K}$ is diagonally dominant. Now, if there is at least one essential boundary condition (say row $J$ ) then $\left|K_{J J}\right|>\rho_{J}$ for the $J$ th row, and hence the matrix is invertible. This establishes the positive-definiteness of $\mathbf{K}$ ( $M$-matrix) and the solvability of the discrete system.

3.1.5. Discrete maximum principle. The continuous problem (diffusion equation) satisfies the maximum principle and hence for stability any discrete approximation for the diffusion operator must also obey the maximum principle.

\section{Theorem}

If $\mathscr{L}_{h} u \geqslant 0$ on a region, then the maximum value of $u$ on this region is attained on the boundary. Similarly, if $\mathscr{L}_{h} u \leqslant 0$, then the minimum value of $u$ is attained on the boundary [64].

\section{Proof}

Consider the region $A_{I}$ (Voronoi cell of node $I$ ). Then, on using Equation (20), we can write

$$
\left(\mathscr{L}_{h} u\right)_{I} \geqslant 0 \Rightarrow \frac{1}{A_{I}}\left[\sum_{J=1}^{n} \beta_{I J} u_{J}-\beta_{I} u_{I}\right] \geqslant 0
$$

and since $A_{I}>0$, we have

$$
\sum_{J=1}^{n} \beta_{I J} u_{J}-\beta_{I} u_{I} \geqslant 0
$$

or

$$
\beta_{I} u_{I} \leqslant \sum_{J=1}^{n} \beta_{I J} u_{J}
$$

and since $\beta_{I}>0(\kappa>0)$, we can write

$$
u_{I} \leqslant \sum_{J=1}^{n} \Phi_{I J} u_{J}, \quad \Phi_{I J}=\frac{\beta_{I J}}{\beta_{I}}
$$


In the above equation, $\beta_{I}=\sum_{J} \beta_{I J}$, the $\Phi_{I J}$ form a partition of unity and $0<\Phi_{I J}<1 \forall J$. The right-hand side of Equation (64) is a convex or barycentric (weights sum to unity) combination (for an $n$-gon) of its neighbours. The barycentric sum is always bounded between the minimum and maximum of its coefficients, and since node $I$ is in the interior of the polygon, it follows that

$$
\begin{aligned}
\min _{J} u_{J} & \leqslant \sum_{J=1}^{n} \Phi_{I J} u_{J} \leqslant \max _{J} u_{J} \\
u_{I} & \leqslant \sum_{J=1}^{n} \Phi_{I J} u_{J}
\end{aligned}
$$

and hence an interior node can be a (local) maximum only if all its neighbours have the same maximum value. This completes the proof for the case $\mathscr{L}_{h} u \geqslant 0$. If $\mathscr{L}_{h} u \leqslant 0$, we have

$$
\begin{gathered}
\min _{J} u_{J} \leqslant \sum_{J=1}^{n} \Phi_{I J} u_{J} \leqslant \max _{J} u_{J} \\
\sum_{J=1}^{n} \Phi_{I J} u_{J} \leqslant u_{I}
\end{gathered}
$$

and we now reach the conclusion that an interior node can be a (local) minimum only if all its neighbours have the same minimum value. This completes the proof for both cases.

\section{Remark}

If $\mathscr{L}$ is the Laplacian $(\kappa \equiv 1)$, then $\Phi_{I J} \equiv \phi_{I J}$, where $\phi_{I J}$ is the Laplace shape function for node $J$ evaluated at $\mathbf{x}_{I}$.

3.1.6. Regular grids. Rectangular and hexagonal grids are used in finite difference methods. In lattice models, Monte Carlo simulations are typically carried out on square and triangular lattices (hexagonal grid) in which periodic boundary conditions are assumed.

\section{Claim 1}

The Voronoi cell finite difference approximation for the Laplacian reduces to the classical finite difference method for rectangular and hexagonal grids.

\section{Proof}

The first part is shown for a rectangular grid with nodal spacings $h_{1}$ and $h_{2}$ in the co-ordinate directions (Figure 7). Using Equation (20) and noting that $\beta_{I J}=\alpha_{I J}(\kappa \equiv 1)$, we can write the finite difference approximation for the Laplacian $\left(\mathscr{L}_{h}=\nabla_{h}^{2}\right)$ at node $I$ as

$$
\nabla_{h}^{2} u=\frac{1}{A_{I}}\left[\sum_{J=1}^{4} \alpha_{I J} u_{J}-\alpha_{I} u_{I}\right], \quad \alpha_{I J}=\frac{s_{I J}}{h_{I J}}, \quad \alpha_{I}=\sum_{J=1}^{4} \alpha_{I J}
$$

From Figure 7, we note that $A_{I}=h_{1} h_{2}, s_{I J}=h_{2}, h_{I J}=h_{1}(J=1,2), s_{I J}=h_{1}, h_{I J}=h_{2}(J=3,4)$, and hence $s_{I J} / h_{I J}=h_{2} / h_{1}(J=1,2)$ and $s_{I J} / h_{I J}=h_{1} / h_{2}(J=3,4)$. The above equation reduces to

$$
\nabla_{h}^{2} u=\frac{1}{h_{1} h_{2}}\left[\frac{h_{2}}{h_{1}}\left(u_{1}+u_{2}\right)+\frac{h_{1}}{h_{2}}\left(u_{3}+u_{4}\right)-2\left(\frac{h_{1}}{h_{2}}+\frac{h_{2}}{h_{1}}\right) u_{I}\right]
$$


which simplifies to

$$
\nabla_{h}^{2} u=\frac{u_{1}+u_{2}-2 u_{I}}{h_{1}^{2}}+\frac{u_{3}+u_{4}-2 u_{I}}{h_{2}^{2}}
$$

which is the five-point finite difference approximation for the Laplacian on a rectangular grid. If $h_{1}=h_{2}=h$, then

$$
\nabla_{h}^{2} u=\frac{u_{1}+u_{2}+u_{3}+u_{4}-4 u_{I}}{h^{2}}
$$

which is the well-known five-point stencil for the Laplacian on a square grid. The finite difference weights for a square grid are shown in Figure 8(a).

Consider the hexagonal grid shown in Figure 8(b). Referring to Equation (67), we can once again write the finite difference approximation for the Laplacian at node $I$ as

$$
\nabla_{h}^{2} u=\frac{1}{A_{I}}\left[\sum_{J=1}^{6} \alpha_{I J} u_{J}-\alpha_{I} u_{I}\right], \quad \alpha_{I J}=\frac{s_{I J}}{h_{I J}}, \quad \alpha_{I}=\sum_{J=1}^{6} \alpha_{I J}
$$

For a hexagonal grid, $h_{I J}=h, s_{I J}=s$, and hence $\alpha_{I J}=s / h \forall J$. In addition, using Equation (19b), we have $A_{I}=6 \mathrm{sh} / 4$. Hence, the above equation can be written as

$$
\nabla_{h}^{2} u=\frac{2}{3 s h}\left[\sum_{J=1}^{6} \frac{s}{h} u_{J}-\frac{6 s}{h} u_{I}\right]
$$

and therefore

$$
\nabla_{h}^{2} u=\frac{2}{3 h^{2}}\left(u_{1}+u_{2}+u_{3}+u_{4}+u_{5}+u_{6}-6 u_{I}\right)
$$

which is the seven-point stencil (hexagonal grid) for the finite difference method. The finite difference weights for this case are shown in Figure 8(b). This completes the proof.

3.1.7. Patch test. The proposed finite difference scheme exactly satisfies the Laplace equation with linear Dirichlet boundary conditions (patch test).

\section{Claim 2}

The Voronoi cell finite difference approximation passes the patch test for any nodal discretization.

\section{Proof}

Consider a domain $\Omega$ that is discretized by $M$ nodes. Let $I$ be an interior node with $n$ neighbours. Then, using Equation (20), the equation for the $I$ th node can be written as $\left(\nabla_{h}^{2} u=0\right)$ :

$$
\frac{1}{A_{I}}\left[\sum_{J=1}^{n} \alpha_{I J} u_{J}-\alpha_{I} u_{I}\right]=0
$$

or

$$
u_{I}=\sum_{J=1}^{n} \phi_{I J} u_{J}, \quad \phi_{I J}=\frac{\alpha_{I J}}{\alpha_{I}}, \quad \alpha_{I J}=\frac{s_{I J}}{h_{I J}}, \quad \alpha_{I}=\sum_{J=1}^{n} \alpha_{I J}
$$


If $I$ is the only interior node (all $J$ are on the boundary), and $u_{J}=a_{0}+a_{1} x_{J}+a_{2} y_{J}$ is the Dirichlet boundary condition (linear field), then

$$
u_{I}=\sum_{J=1}^{n} \phi_{I J}\left(a_{0}+a_{1} x_{J}+a_{2} y_{J}\right)=a_{0}+a_{1} x_{I}+a_{2} y_{I}
$$

since the $\phi_{I J}$ form a partition of unity and they also satisfy the linear reproducing conditions given in Equation (11). Hence the solution at an interior node is identical to the exact solution. By a similar extension, if there are more nodes in the interior of the domain, all equations for $u_{I}$ that arise are similar to Equation (75) and can hence reproduce the linear field exactly. A consequence of the above is that the solution $u_{I}$ at all interior nodes $I$ is identical to the exact solution (linear field) evaluated at $\mathbf{x}_{I}$, which completes the proof.

\section{NUMERICAL EXAMPLES}

Numerical examples for the finite difference scheme on Voronoi grids are presented to demonstrate the accuracy and convergence of the scheme.

\subsection{Finite-difference scheme on arbitrary grids}

Numerical examples are presented for the 2D Poisson equation with Dirichlet boundary conditions. The model problem is:

$$
\begin{aligned}
-\nabla^{2} u(\mathbf{x})=f(\mathbf{x}) \quad \text { in } \Omega \\
u(\mathbf{x})=g(\mathbf{x}) \quad \text { on } \partial \Omega
\end{aligned}
$$

First we show that the finite difference scheme passes the patch test on uniform as well as non-uniform grids. Then, convergence studies in the $L^{2}$ norm are carried out for three test cases to demonstrate the accuracy and convergence of the method. Lastly, numerical results for the Laplace equation in an annulus are presented to demonstrate the performance of the scheme on non-rectangular domains. In our analysis, the $L^{2}$ and $L^{\infty}$ (maximum) discrete norms are defined as

$$
\left\|u-u_{h}\right\|_{2, \Omega}=\sqrt{A_{I} \sum_{I=1}^{M}\left(u\left(\mathbf{x}_{I}\right)-u_{h}\left(\mathbf{x}_{I}\right)\right)^{2}}, \quad\left\|u-u_{h}\right\|_{\infty, \Omega}=\max _{I=1, \ldots, M}\left|u\left(\mathbf{x}_{I}\right)-u_{h}\left(\mathbf{x}_{I}\right)\right|
$$

where $u$ and $u_{h}$ are the exact and the finite difference solutions, respectively, and $A_{I}$ is the area of the Voronoi cell of node $I$. The discrete $L^{2}$ norm in Equation (78) is defined analogous to its continuous counterpart- $\|u\|_{L^{2}(\Omega)}=\left(\int_{\Omega} u^{2} \mathrm{~d} \Omega\right)^{1 / 2}$.

4.1.1. Patch test. The patch test for the Laplacian operator is carried out: $\nabla^{2} u=0$ in $\Omega=$ $(0,1) \times(0,1)$, with $u=g(\mathbf{x})=x+y$ (linear field) imposed on the boundary of the unit square. The exact solution for this problem is: $u(\mathbf{x})=x+y$. If the numerical scheme passes the patch test, then the solution $u_{I}$ at interior nodes must be in agreement (within machine precision) with the exact solution. 

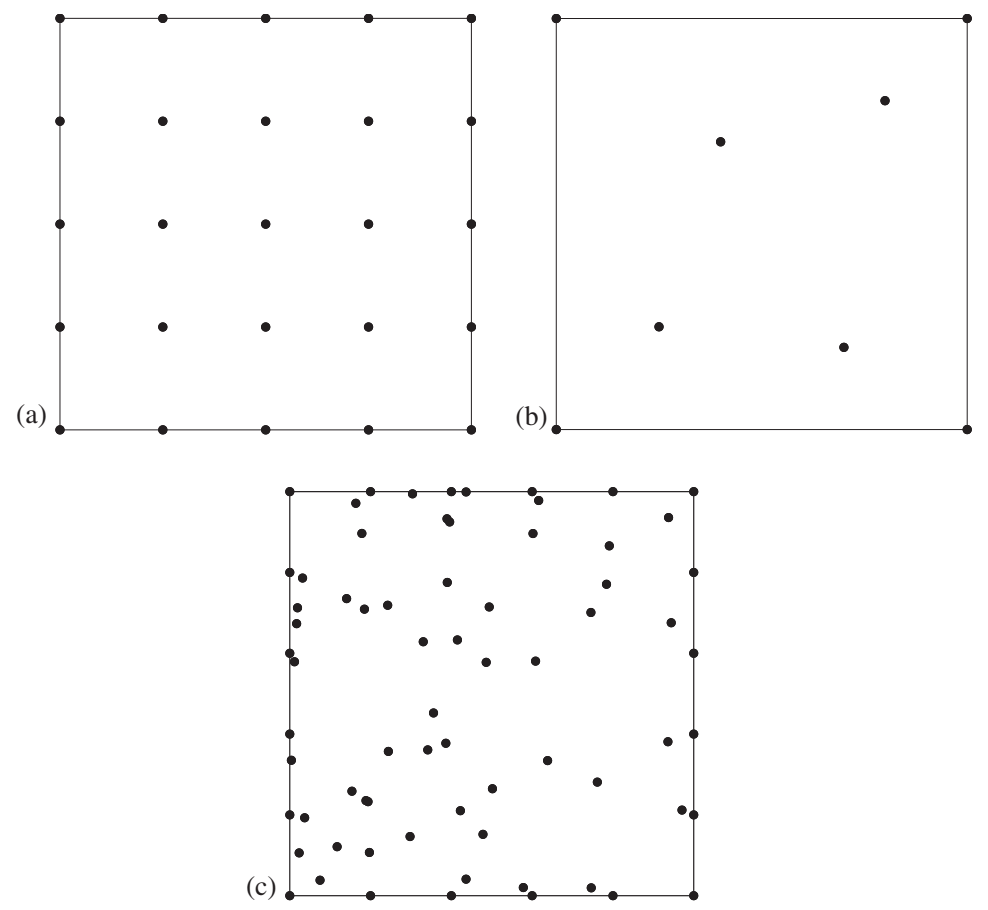

Figure 9. Patch test: (a) uniform grid (25 nodes); (b) irregular grid (8 nodes); and (c) Random set (70 nodes).

Table I. Errors in the $L^{2}$ and $L^{\infty}$ norms for the patch test.

\begin{tabular}{lcc}
\hline & $\frac{\left\|u-u_{h}\right\|_{2, \Omega}}{\|u\|_{2, \Omega}}$ & $\left\|u-u_{h}\right\|_{\infty, \Omega}$ \\
\hline arids & $4.9 \times 10^{-17}$ & $2.2 \times 10^{-16}$ \\
b & $3.8 \times 10^{-17}$ & $1.1 \times 10^{-16}$ \\
c & $2.0 \times 10^{-16}$ & $7.5 \times 10^{-16}$ \\
\hline
\end{tabular}

Three different nodal discretizations [29] are considered (Figure 9), and the relative error in the $L^{2}$ norm and the maximum norm are presented in Table I. The results in Table I indicate that the patch test is passed for regular as well as irregular grids, and hence the numerical tests do confirm the proof outlined in Section 3.1.7.

4.1.2. Poisson equation in a unit square. Convergence in the $L^{2}$ norm is studied for the proposed finite-difference scheme. To this end, three Poisson problems are solved in a unit square $(\Omega=(0,1) \times(0,1))$. The boundary conditions, forcing function, and exact solution for the selected examples are given in Table II. In order to conduct the convergence study, 
Table II. Numerical tests for the Poisson problem in a unit square.

\begin{tabular}{lccc}
\hline Problem & $f(\mathbf{x})$ & $g(\mathbf{x})$ & $u(\mathbf{x})$ \\
\hline I & $2 x(1-x)+2 y(1-y)$ & 0 & $x(1-x) y(1-y)$ \\
II & $2 \pi^{2} \sin \pi x \sin \pi y$ & 0 & $\sin \pi x \sin \pi y$ \\
III & 0 & $\begin{array}{c}\sin \pi x \sinh \pi \text { on } y=1 ; \\
0 \text { otherwise }\end{array}$ & $\sin \pi x \sinh \pi y$ \\
& &
\end{tabular}

a Delaunay triangulation mesh generator is used so that uniform grids for a user-specified desired value of $h$ (grid spacing) are obtained. The mesh generator is based on the algorithm developed in Reference [72], in which the initial boundary conforming triangulation is carried out using the Tanemura-Ogawa-Ogita algorithm [73] and subsequent refinement is based on incremental point insertion using the Bowyer-Watson algorithm [47,48]. Refinement is based on comparing an actual local length scale $\ell$ (e.g. element width, circum-radius) to the desired length scale specified by a scalar variable which is known as the length density function $\rho$ [72]. In the present instance, since a uniform spacing $h$ is desired in the domain, we set $\rho=h$ (a constant) on all the four sides of the unit square.

For the convergence analysis, four grids are considered: $h=0.1,0.05,0.025,0.0125$. The nodal grids are shown in Figure 10. The spacing is nearly uniform in the entire domain with the number of nodes increasing by about a factor of four at every refinement step. The results of the convergence analysis are presented in Figure 11. The error in the discrete $L^{2}$ norm varies as $h^{\lambda}$, where $\lambda$ is the rate of convergence. It is seen from Figure 11 that the error decreases uniformly with decreasing $h$ and the finite difference method has a rate of convergence of two in the $L^{2}$ norm. In Figure 12, the numerical solution $\left(u_{h}\right)$ and the error $\left(u-u_{h}\right)$ on the unit square (1929 nodal grid) are shown for test problem III. It is observed that the errors are the most pronounced near $y=1$. The maximum (absolute) error is $2.6 \times 10^{-3}$, where the exact solution is of the order of 10 (relative error is $0.03 \%$ ).

4.1.3. Poisson equation in an annulus. To demonstrate the accuracy of the proposed technique on non-rectangular domains, we solve the Poisson equation in an annulus which is bounded between two concentric cylinders of radius $r=1$ and 2:

$$
\begin{aligned}
-\nabla^{2} u(r, \theta) & =f(r, \theta) \quad \text { in } 1<r<2 \\
u(1, \theta) & =\bar{u}_{1}(\theta), \quad u(2, \theta)=\bar{u}_{2}(\theta)
\end{aligned}
$$

which in electrostatics is the model problem for the potential in the presence of a charge distribution in the domain $-u$ is the potential, $f$ is the charge density, and $\mathbf{E}=-\nabla u$ (flux) is the electric field (force per unit charge).

Numerical tests are presented for three problems:

I. The Laplace equation (no charges) with the potential a constant on both the inner and the outer boundaries $\left(\bar{u}_{1}=0, \bar{u}_{2}=1\right)$ is considered. Owing to symmetry, the Laplace 
(a)

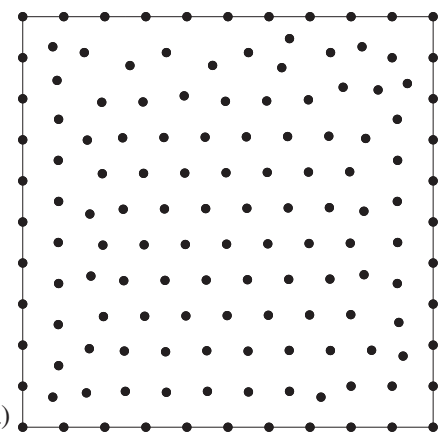

(c)

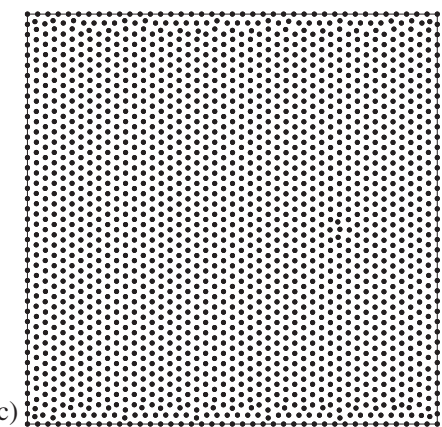

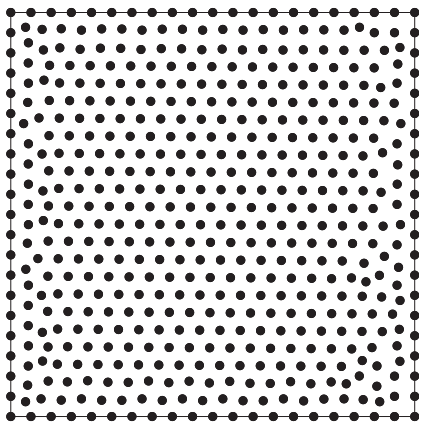

(d)

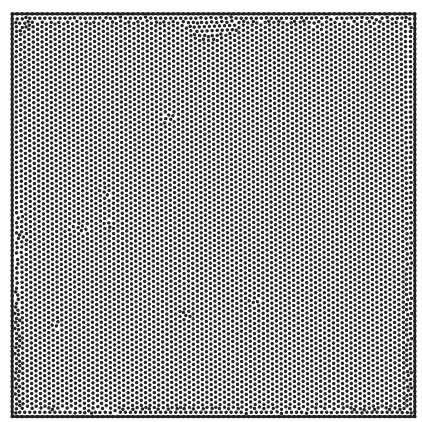

Figure 10. Nodal grids for the Poisson problems in a unit square: (a) 138 nodes;

(b) 509 nodes; (c) 1929 nodes; and (d) 7542 nodes.

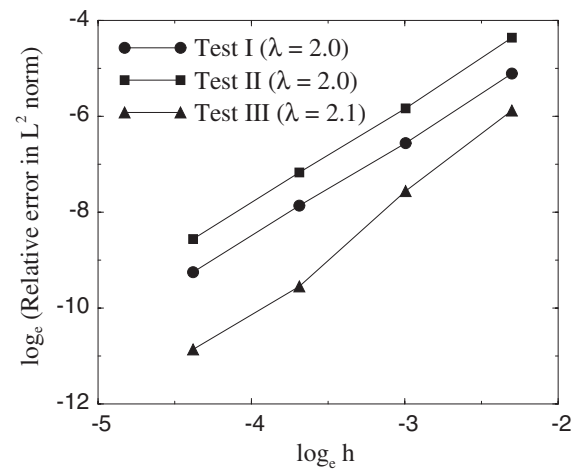

Figure 11. Rate of convergence for Poisson problems in a unit square.

The convergence rate $\lambda$ is indicated.

equation reduces to the $1 \mathrm{D}$ radial equation: $u_{r r}+u_{r} / r=0$ with the exact solution:

$$
u(r)=\frac{\ln (r)}{\ln (2)}
$$

II. As a second test, we consider the Laplace equation with boundary conditions that vary with $\theta: \bar{u}_{1}=1+\cos \theta$, and $\bar{u}_{2}=2+\cos \theta$. The exact solution using separation of variables 

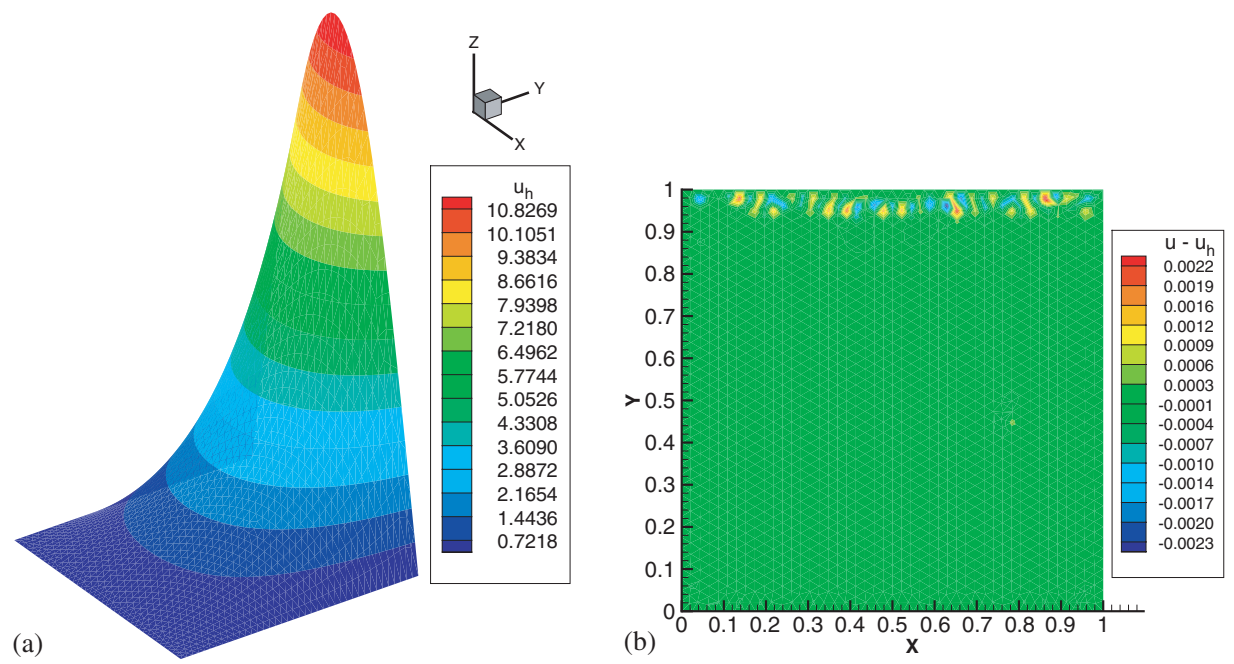

Figure 12. Numerical solution and error for test problem III: (a) $u_{h}$; and (b) $u-u_{h}$.

can be written as

$$
u(r, \theta)=1+\frac{\ln (r)}{\ln (2)}+\left[\frac{r}{3}+\frac{2}{3 r}\right] \cos \theta
$$

III. Lastly, the Poisson problem with a charge distribution $f(r, \theta)=-\left(15 r^{2}-24 r+6\right) \sin \theta$ and with homogeneous boundary conditions $\bar{u}_{1}=\bar{u}_{2}=0$ is studied. The exact solution is given by [74]

$$
u(r, \theta)=\left(r^{4}-3 r^{2}+2 r^{2}\right) \sin \theta
$$

Four nodal grids are used to carry out a convergence study. In generating the nodal grids, a constant value of $\rho=h$ is specified on both the inner radius $(r=1)$ as well as the outer radius $(r=2)$. The nodal grids used in the analysis are shown in Figure 13 with the average grid spacing $h=\pi / 8, \pi / 16, \pi / 32$, and $\pi / 64$. The relative error in the $L^{2}$ norm is plotted in Figure 14, and it is seen that even for a non-rectangular domain, the second-order accuracy of the method is preserved. In Figure 15, the numerical solution for the finest grid is plotted. The symmetry in the potential structure is captured, and the maximum error in the domain (point-wise estimate at the nodes) is $0.4 \%$.

\section{CONCLUSIONS}

In this study, we have presented some of our recent findings on the Laplace interpolant and have provided a basis for its use as a suitable weight measure for unstructured grids. The Voronoi diagram and the notion of natural neighbours were used as the building blocks in describing the link between discrete nodes on an irregular grid. A detailed account of the 

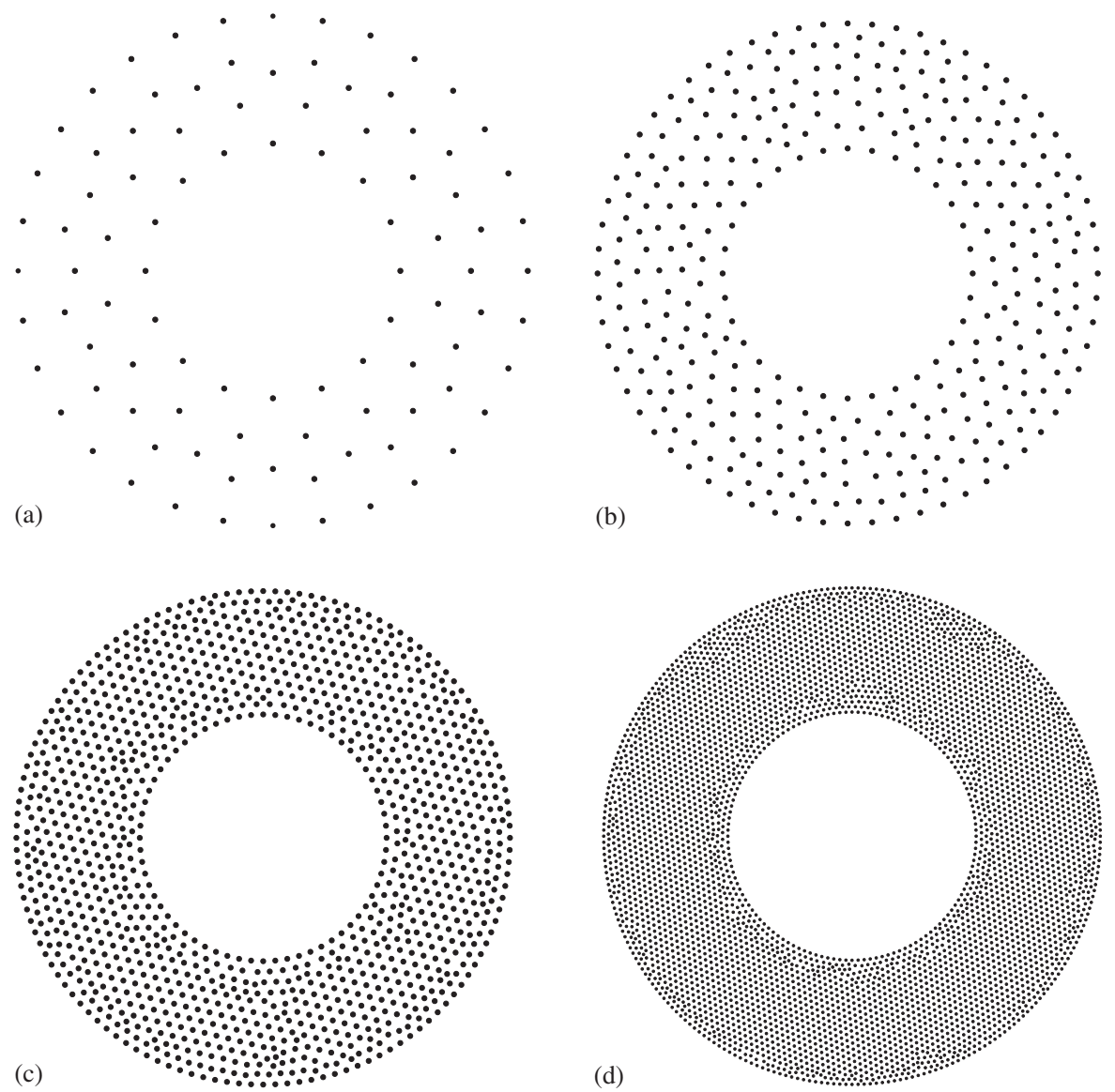

Figure 13. Nodal grids for the Poisson problems in an annulus: (a) 96 nodes;

(b) 346 nodes; (c) 1257 nodes; and (d) 4744 nodes.

previous developments of the Laplace interpolant was presented, and then the Laplace weight was used to construct a robust and accurate Voronoi cell finite difference method for arbitrary unstructured grids.

The Voronoi cell finite difference scheme that was proposed was distinctive in many respects; some of the key advantages of the numerical method were as follows:

1. Liszka and Orkisz [3] recognized the importance of spatial nodal arrangements and appropriate geometries of the computational cell that rendered well-conditioned and solvable system of equations. Natural neighbours present a very appealing construct in defining neighbour relations on an arbitrary grid, and hence it is evident that using this notion in the construction of a discrete approximation is of merit. In addition, as opposed to earlier approaches which rely on Euclidean distances to define the computational cell for a node, here the use of the Voronoi polygon was a natural choice. 


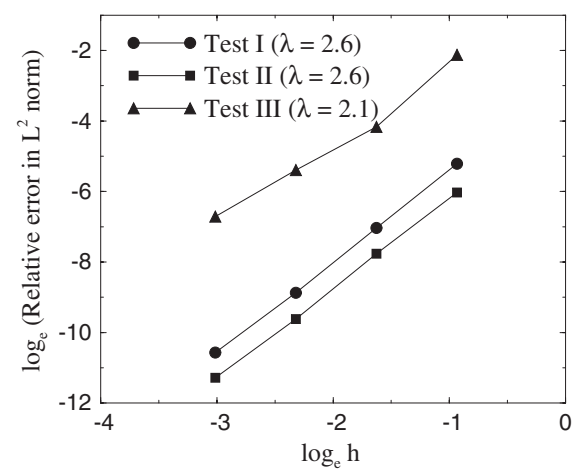

Figure 14. Rate of convergence for the Poisson problems in an annulus.

The convergence rate $\lambda$ is indicated.

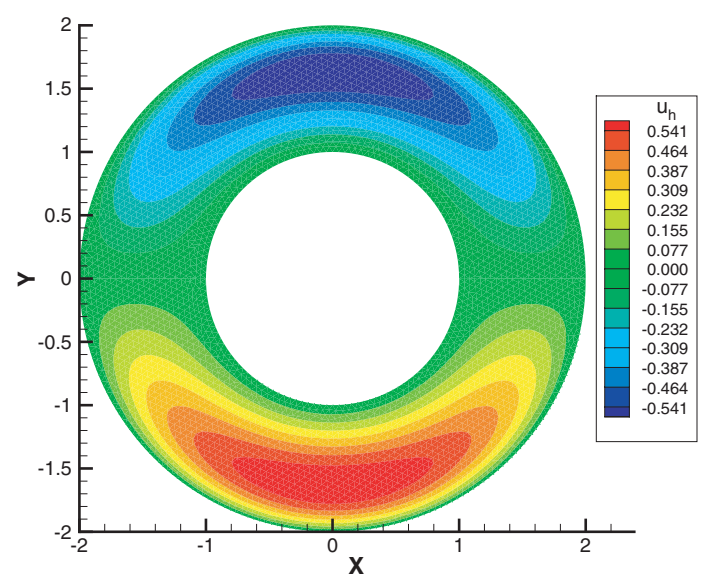

Figure 15. Numerical solution for the Poisson problem with a non-zero charge distribution.

2. The finite difference approximation was derived from a conservation principle for the diffusion equation and hence a link between the discrete approximation and the differential equation was established. Conservation laws play a prominent role in the formulation of finite volume schemes, and their use in constructing finite difference schemes is also of merit [75].

3. The use of the Laplace weights provides a simple means to obtain the finite difference weights for the Laplace operator on any 1D grid.

4. Solutions that satisfy the diffusion equation (for instance, Laplace or Poisson equation) obey the maximum principle, and it was shown in Section 3.1.5 that the finite difference approximation also inherits this property for any unstructured grid. A detailed study on consistency using a Taylor series analysis revealed that the finite difference scheme was only zeroth-order consistent on irregular grids. This leads us to previous studies on finite volume schemes on non-uniform grids, the notion of supraconvergence, and to prior 
theoretical work $[8,10]$ to provide support for the convergence of the method. The use of a balance law (flux conservation) to construct the difference scheme, in tandem with the satisfaction of the patch test and the added stability lead to second-order convergence (supraconvergence) for the problems considered in this study.

5. The diffusion operator is self-adjoint, and the discrete diffusion operator results in a symmetric positive-definite stiffness matrix. This was a consequence of the symmetry of the Laplace weight $\left(\alpha_{I J}=\alpha_{J I}\right)$. Most finite difference and finite volume schemes for the diffusion (Laplace) operator on irregular grids yield a non-symmetric matrix. If the Sibson shape function or those based on MLS schemes are used as weights in a finite difference scheme, in general a non-symmetric stiffness matrix would result.

6. Only simple geometric computations are required to evaluate the finite difference weighting functions, and hence the computational costs involved in the finite difference scheme are minimal. In 3D, the only additional complexity that arises is in the computation of $s_{I J}$ which is a polygonal area (Voronoi facet) [38].

The numerical simulations in this paper have demonstrated the promise of the Laplace weight as an appropriate measure on unstructured grids. The use of a local conservation principle to derive the finite difference (or equivalently collocation) scheme is of relevance in nodal integration techniques within meshless methods [60], and also in hydrodynamic simulations using particle methods where local conservation is desirable [76]. On considering integral identities and rules of vector algebra on lattices networks, a general framework can possibly emerge for the construction of finite difference approximations on unstructured grids that involve the gradient and divergence of vectors as well as higher-order tensors.

\section{ACKNOWLEDGEMENTS}

It is a pleasure to acknowledge John Bolander and Tim Ginn for many stimulating discussions, and for their comments on an earlier draft of this paper; the author is also grateful to Tim Ginn for pointing out the related work in Reference [58]. Parts of this study were completed during the spring of 2001, when the author was at the Princeton Materials Institute; the hospitality extended to him by David Srolovitz at Princeton University is appreciated. Tim Baker is also thanked for providing the Delaunay triangulation code, which was used to generate the grids considered in the convergence study.

\section{REFERENCES}

1. Belytschko, T, Krongauz, Y, Organ, D, Fleming, M, Krysl P. Meshless methods: an overview and recent developments. Computer Methods in Applied Mechanics and Engineering 1996; 139:3-47.

2. Jensen PS. Finite difference techniques for variable grids. Computers and Structures 1972; 2:17-29.

3. Liszka T, Orkisz J. The finite difference method at arbitrary irregular grids and its application in applied mechanics. Computers and Structures 1980; 11:83-95.

4. Breitkopf P, Touzot G, Villon P. Double grid diffuse collocation method. Computational Mechanics 2000; 25(2/3):199-206.

5. Lancaster P, Salkauskas K. Surfaces generated by moving least squares methods. Mathematics of Computation 1981; 37:141-158.

6. Baty RS, Villon WP. Least-squares solutions of a general numerical method for arbitrary irregular grids. International Journal for Numerical Methods in Engineering 1997; 140:1701-1717.

7. Süli E. Convergence of finite volume schemes for Poisson's equations on nonuniform meshes. SIAM Journal on Numerical Analysis 1991; 28(5):1419-1430.

8. Mishev ID. Finite volume methods on Voronoi meshes. Numerical Methods for Partial Differential Equations 1998; 14:193-212.

9. Hermeline F. A finite volume method for the approximation of diffusion operators on distorted meshes. Journal of Computational Physics 2000; 160:481-499. 
10. Jones WP, Menzies KR. Analysis of the cell-centred finite volume method for the diffusion equation. Journal of Computational Physics 2000; 165:45-68.

11. Petrovskaya NB. Modification of a finite volume scheme for Laplace's equation. SIAM Journal on Scientific Computing 2001; 23(3):891-909.

12. Christie I, Hall C. The maximum principle for bilinear elements. International Journal for Numerical Methods in Engineering 1984; 20:549-553.

13. Putti M, Cordes C. Finite element approximation of the diffusion operator on tetrahedra. SIAM Journal on Scientific Computing 1998; 19(4):1154-1168.

14. Li S, Liu WK. Meshfree and particle methods and their applications. Applied Mechanics Reviews 2002; 55(1): $1-34$.

15. Melenk JM, Babuška I. The partition of unity finite element method: Basic theory and applications. Computer Methods in Applied Mechanics and Engineering 1996; 139:289-314.

16. Duarte CA, Oden JT. An $H$ - $p$ adaptive method using clouds. Computer Methods in Applied Mechanics and Engineering 1996; 139:237-262.

17. Sibson R. A vector identity for the Dirichlet tessellation. Mathematical Proceedings of the Cambridge Philosophical Society 1980; 87:151-155.

18. Sibson R. A brief description of natural neighbor interpolation. In Interpreting Multivariate Data, Barnett V (ed.). John Wiley: Chichester, 1981; 21-36.

19. Okabe A, Boots B, Sugihara K. Spatial Tessellations: Concepts and Applications of Voronoi Diagrams. Wiley: Chichester, 1992.

20. Bern M, Eppstein D. Mesh generation and optimal triangulation. In Computing in Euclidean Geometry, Du D-Z, Hwang FK (eds). Lecture Notes Series on Computing, vol. 1. World Scientific: Singapore, 1995; 23-90.

21. Belikov VV, Ivanov VD, Kontorovich VK, Korytnik SA, Semenov AYu. The non-Sibsonian interpolation: A new method of interpolation of the values of a function on an arbitrary set of points. Computational Mathematics and Mathematical Physics 1997; 37(1):9-15.

22. Belikov VV, Semenov AYu. New non-Sibsonian interpolation on arbitrary system of points in Euclidean space. In 15th IMACS World Congress, Numerical Mathematics, vol. 2. Wissen Tech. Verlag: Berlin, 1997; $237-242$.

23. Belikov VV, Semenov AYu. Non-Sibsonian interpolation on arbitrary system of points in Euclidean space and adaptive isolines generation. Applied Numerical Mathematics 2000; 32(4):371-387.

24. Sugihara K. Surface interpolation based on new local coordinates. Computer-Aided Design 1999; 31:51-58.

25. Hiyoshi H, Sugihara K. Two generalizations of an interpolant based on Voronoi diagrams. International Journal of Shape Modeling 1999; 5(2):219-231.

26. Hiyoshi H, Sugihara K. Voronoi-based interpolation with higher continuity. In Proceedings of the 16th Annual ACM Symposium on Computational Geometry, 2000; 242-250.

27. Braun J, Sambridge M. A numerical method for solving partial differential equations on highly irregular evolving grids. Nature 1995; 376:655-660.

28. Sukumar N. The natural element method in solid mechanics. Ph.D. Thesis, Theoretical and Applied Mechanics, Northwestern University, Evanston, IL, U.S.A., June 1998.

29. Sukumar N, Moran B, Belytschko T. The natural element method in solid mechanics. International Journal for Numerical Methods in Engineering 1998; 43(5):839-887.

30. Sukumar N, Moran B. $C^{1}$ natural neighbor interpolant for partial differential equations. Numerical Methods for Partial Differential Equations 1999; 15(4):417-447.

31. Bueche D, Sukumar N, Moran B. Dispersive properties of the natural element method. Computational Mechanics 2000; 25(2/3):207-219.

32. Sukumar N, Moran B, Semenov AYu, Belikov VV. Natural neighbor Galerkin methods. International Journal for Numerical Methods in Engineering 2001; 50(1):1-27.

33. Sukumar N. Sibson and non-Sibsonian interpolants for elliptic partial differential equations. In Proceedings of the First MIT Conference on Fluid and Solid Mechanics, vol. 2, Bathe KJ (ed.). Elsevier Press: Amsterdam, The Netherlands, 2001; 1665-1667.

34. Cueto E, Doblaré M, Gracia L. Imposing essential boundary conditions in the natural element method by means of density-scaled $\alpha$-shapes. International Journal for Numerical Methods in Engineering 2000; 49(4):519-546.

35. García JM, Cueto E, Doblaré M. Simulation of bone internal remodeling by means of the $\alpha$-shape-based natural element method. In European Congress on Computational Methods in Applied Sciences and Engineering, ECCOMAS 2000, Barcelona, Spain, 2000.

36. Moran B, Yoo J. Meshless methods for life cycle engineering simulation: natural neighbor formulations. In Modeling and Simulation-Based Life Cycle Engineering Simulation, Chong KP, Saigal S, Thynell S, Morgan H (eds). Taylor and Francis: London, 2001; 91.

37. Cueto E, Calvo B, Doblaré M. Modelling three-dimensional piece-wise homogeneous domains using the $\alpha$-shape-based natural element method. International Journal for Numerical Methods in Engineering 2002; 54(6):871-897. 
38. Sukumar N, Bolander Jr. JE. Numerical computation of discrete differential operators on non-uniform grids. 2002, submitted.

39. Martinez MA, Cueto E, Doblaré M, Chinesta F. Natural element meshless simulation of injection processes involving short fiber suspension. Journal of Non-Newtonian Fluid Mechanics, 2003, in press.

40. Christ NH, Friedberg R, Lee TD. Random lattice field-theory-general formulation. Nuclear Physics B 1982; 202(1):89-125.

41. Christ NH, Friedberg R, Lee TD. Gauge-theory on a random lattice. Nuclear Physics B 1982; 210(3):310-336.

42. Christ NH, Friedberg R, Lee TD. Weights of links and plaquettes in a random lattice. Nuclear Physics B 1982; 210(3):337-346.

43. Belytschko T, Lu YY, Gu L. Element-free Galerkin methods. International Journal for Numerical Methods in Engineering 1994; 37:229-256.

44. Yavari A, Kaveh A, Sarkani S, Bondarabady HAR. Topological aspects of meshless methods and nodal ordering for meshless discretizations. International Journal for Numerical Methods in Engineering 2001; 52(9): 939-978.

45. Lawson CL. Software for $C^{1}$ surface interpolation. In Mathematical Software III, vol. 3, Rice JR (ed.). Academic Press: New York, NY, 1977.

46. Grünbaum B. Convex Polytopes. Wiley: New York, 1967.

47. Bowyer A. Computing Dirichlet tessellations. The Computer Journal 1981; 24:162-166.

48. Watson DF. Computing the $n$-dimensional Delaunay tessellation with application to Voronoi polytopes. The Computer Journal 1981; 24(2):167-172.

49. Beale PD, Srolovitz DJ. Elastic fracture in random materials. Physics Review B 1988; 37(10):5500-5507.

50. Jagota A, Bennison SJ. Spring-network and finite-element models for elasticity and fracture. In Nonlinearity and Breakdown in Soft Condensed Matter. Springer Lecture Notes in Physics, vol. 437, Bardhan KK, Chakrabarti BK, Hansen A (eds). Springer: Berlin, 1994; 186-201.

51. Jagota A, Bennison SJ. Element breaking rules in computational models for brittle fracture. Modelling and Simulation in Materials Science and Engineering 1995; 3(4):485-501.

52. Bolander Jr JE, Saito S. Fracture analyses using spring networks with random geometry. Engineering Fracture Mechanics 1998; 61:569-591.

53. Bolander Jr JE, Yoshitake K, Thomure J. Stress analysis using elastically uniform rigid-body spring networks. Journal of Structural Mechanics and Earthquake Engineering 1999; 633/I-49:25-32.

54. Harris SC, Williams D, Sibson R. Scaling random walks on arbitrary sets. Mathematical Proceedings of the Cambridge Philosophical Society 1999; 125(3):535-544.

55. Ghosh S, Mukhopadhyay SN. A material based finite-element analysis of heterogeneous media involving Dirichlet tessellations. Computer Methods in Applied Mechanics and Engineering 1993; 104(2):211-247.

56. Ghosh S, Moorthy S. Elastic-plastic analysis of arbitrary heterogeneous materials with the Voronoi cell finiteelement method. Computer Methods in Applied Mechanics and Engineering 1995; 121(1-4):373-409.

57. Friedberg R, Ruiz M. Vector algebra on a lattice. Physical Review D 1984; 29(12):2916-2918.

58. Narasimhan TN, Witherspoon PA. An integrated finite difference method for analyzing fluid flow in porous media. Water Resources Research 1976; 12(1):57-64.

59. Aluru NR. A point collocation method based on reproducing kernel approximations. International Journal for Numerical Methods in Engineering 2000; 47:1083-1121.

60. Chen JS, Wu CT, Yoon S, You Y. A stabilized conforming nodal integration for Galerkin meshfree methods. International Journal for Numerical Methods in Engineering 2001; 50:435-466.

61. Börgers C, Peskin CS. A Lagrangian method based on the Voronoi diagram for the incompressible NavierStokes equations on a periodic domain. In The Free Lagrange Method (Lecture Notes in Physics), vol. 238, Frills MJ, Crowley WP, Trease H (eds). Springer-Verlag: New York, 1985; 87-113.

62. Börgers C, Peskin CS. A Lagrangian fractional step method for the incompressible Navier-Stokes equations on a periodic domain. Journal of Computational Physics 1987; 70:397-438.

63. Thomas JW. Numerical Partial Differential Equations: Finite Difference Methods. Springer-Verlag: New York, 1995.

64. Strikwerda JC. Finite Difference Schemes and Partial Differential Equations. Chapman \& Hall, CRC: Boca Raton, FL, 1999.

65. Tikhonov AN, Samarskii AA. Homogeneous difference schemes on non-uniform nets. U.S.S.R Computational Mathematics and Mathematical Physics 1962; 2:927-953.

66. Kreiss H-O, Manteuffel TA, Swartz B, Wendroff B, White Jr AB. Supra-convergent schemes on irregular grids. Mathematics of Computation 1986; 47(176):537-554.

67. Manteuffel TA, White Jr. AB. The numerical solution of second-order boundary value problems on nonuniform meshes. Mathematics of Computation 1986; 47(176):511-535.

68. Weiser A, Wheeler MF. On convergence of block-centered finite differences for elliptic problems. SIAM Journal on Numerical Analysis 1998; 25(2):351-375.

69. Lazarov RD, Mishev ID, Vassilevski PS. Finite volume methods for convection-diffusion problems. SIAM Journal on Numerical Analysis 1996; 33(1):31-55. 
70. Cockburn B, Gremaud P-A, Yang JX. A priori error estimates for numerical methods for scalar conservation laws part III: Multidimensional flux-splitting monotone schemes on non-cartesian grids. SIAM Journal on Numerical Analysis 1998; 35(5):1775-1803.

71. Thomas JW. Numerical Partial Differential Equations: Conservation Laws and Elliptic Equations. SpringerVerlag: New York, 1999.

72. Baker TJ. Triangulations, mesh generation and point placement strategies. In Frontiers of Computational Fluid Dynamics, Caughey DA, Hafez MM (eds). Wiley: New York, NY, 1994; 101-115.

73. Tanemura M, Ogawa T, Ogita W. A new algorithm for three-dimensional Voronoi tessellation. Journal of Computational Physics 1983; 51:191-207.

74. Christopher I, Knorr G, Shoueri M, Bertrand P. Solution of the Poisson equation in an annulus. Journal of Computational Physics 1997; 131:323-326.

75. Shashkov M. Conservative Finite-Difference Methods on General Grids. CRC Press: Boca Raton, FL, 1996.

76. Dilts GA. Moving least-square particle hydrodynamics II: Conservation and boundaries. International Journal for Numerical Methods in Engineering 2000; 48:1503-1524. 